\title{
Quantum toric degeneration of quantum flag and Schubert varieties
}

\author{
L. Rigal, P. Zadunaisky
}

\begin{abstract}
We show that certain homological regularity properties of graded connected algebras, such as being AS-Gorenstein or AS-Cohen-Macaulay, can be tested by passing to associated graded rings. In the spirit of noncommutative algebraic geometry, this can be seen as an analogue of the classical result that, in a flat family of varieties over the affine line, regularity properties of the exceptional fiber extend to all fibers. We then show that quantized coordinate rings of flag varieties and Schubert varieties can be filtered so that the associated graded rings are twisted semigroup rings in the sense of [RZ12]. This is a noncommutative version of the result due to Caldero [Calo2] stating that flag and Schubert varieties degenerate into toric varieties, and implies that quantized coordinate rings of flag and Schubert varieties are AS-Cohen-Macaulay.
\end{abstract}

\section{Introduction}

Let $\mathbb{k}$ be a field, and let $A$ be a noetherian commutative algebra over $\mathbb{k}$. If we put an ascending filtration on $A$ then we can build the Rees ring of the filtration, which is a free $\mathbb{k}[t]$-algebra $\mathcal{R}$ such that $A \cong \mathcal{R} /(t-\lambda) \mathcal{R}$ for all $\lambda \in \mathbb{k}^{\times}$, while $\mathcal{R} / t \mathcal{R}$ is isomorphic to the associated graded ring. In geometric terms, if $\mathbb{k}$ is algebraically closed then the variety associated to $\mathcal{R}$ is a flat family over the affine line, whose generic fiber is isomorphic to Spec $A$ and whose fiber over 0 is isomorphic to Spec gr $A$; in this context the fiber over 0 is called a degeneration of $\operatorname{Spec} A$. A standard result from algebraic geometry states that if the fiber over 0 is regular (resp. Gorenstein, Cohen-Macaulay, or any other of a long list of properties) then all fibers are regular (resp. Gorenstein, Cohen-Macaulay, etc.)

Of course, the idea of studying a ring by imposing a filtration and passing to the associated graded ring is a basic tool in an algebraist's toolbox, and can be applied outside of a geometric context. In particular the hypothesis of commutativity is not necessary for filtered-to-graded methods to work. However, in the spirit of noncommutative algebraic geometry, we should look at the case where $A$ is noetherian, $\mathbb{N}$-graded and connected (i.e. $A_{0}=\mathbb{k}$ ) with an eye on the geometric case. Although in this case there are no varieties associated to our algebras as in the commutative setting, we have suitable analogues of the notions of being regular, or Gorenstein, or Cohen-Macaulay, defined in purely homological terms (see paragraph 2.6). Hence it 
makes sense to ask whether these properties are "stable by flat deformation", i.e. if the fact that $\operatorname{gr} A$ has any of these properties implies that $A$ also has that property. The objective of this paper is to develop these ideas in order to study natural classes of noncommutative algebraic varieties, and its first main result 2.8 shows that indeed, good geometric properties are stable by degeneration in this context.

In the commutative setting, the usual flag variety and its Schubert subvarieties are examples where the degeneration method is successful. The same holds for the more general flag and Schubert varieties that one may associate to a semisimple Lie group. On the other hand, the theory of quantum groups provides natural quantum analogues of flag and Schubert varieties, whose classical counterparts can be recovered as semiclassical limits when the deformation parameter tends to 1 . This is the class of noncommutative varieties we intend to study.

The degeneration approach to the study of flag and Schubert varieties was pioneered by de Concini, Eisenbud and Procesi [DCEP82], and followed by Gonciulea and Lakshmibai [GL96], and others. Caldero was the first to prove that any Schubert variety of an arbitrary flag variety degenerates to an affine toric variety in [Calo2]. A degeneration to a toric variety is particularly convenient, since the geometric properties of toric varieties are easily tractable, being encoded in the combinatorial properties of a semigroup naturally attached to them in the affine case. For a general survey on the subject of toric degenerations, including recent results, we refer the reader to [FFLi7a].

It is striking that even though he is interested in the classical objects, in [Calo2] Caldero uses the theory of quantum groups (more precisely the global bases of Luzstig and Kashiwara) to produce bases of the coordinate rings of the classical objects. His main argument then relies on Littelmann's string parametrization of the crystal basis of the negative part of the quantized enveloping algebra, which allows him to show that his bases have good multiplicative properties. It follows that one can build out of them adequate filtrations of the coordinate rings, leading to a toric degeneration.

In contrast, we wish to produce degenerations of quantum Schubert varieties at the noncommutative level. This led us to introduce noncommutative analogues of affine toric varieties as a suitable target for degeneration and to study their geometric properties in [RZ15], where we show that just as in the classical case, these properties are encoded in the associated semigroup. Inspired by Caldero's work, in this article we show that quantum Schubert varieties degenerate into quantum toric varieties and explore the consequences of this fact.

We now discuss the contents of each section.

Section 2 begins with a recollection of general material on connected $\mathbb{N}^{r}$-graded algebras, their homological regularity properties, and the behavior of these properties with respect to a change of grading. In the last subsection we prove our main transfer result: if a connected $\mathbb{N}^{r}$-graded algebra has a filtration by finite dimensional graded subspaces, then (under a mild technical condition known as property $\chi$ ) the original algebra inherits the regularity properties of the associated graded algebra, see Theorem 2.12. The key point is the existence of a spectral sequence relating the Ext 
spaces between an algebra and its associated graded algebra; property $\chi$ guarantees that these spaces are finite dimensional, which is crucial for the transfer of properties.

The aim of section 3 is to introduce a general framework allowing to establish the existence of a noncommutative toric degeneration. We first review the basic theory of affine semigroups and the regularity properties of quantum affine toric varieties from [RZ15]. We then introduce the notion of an algebra of $(S, \varphi)$-type, where $S$ is an affine semigroup $S \subset \mathbb{N}^{r}$ and $\varphi: S \longrightarrow \mathbb{N}$ is a semigroup morphism. Any positive affine semigroup has a unique minimal presentation as a quotient of a finitely generated free noncommutative monoid. An algebra of $(S, \varphi)$-type is an algebra with a presentation modeled after the presentation of $S$, with as many generators as $S$ and whose product reflects that of $S$ up to terms of smaller order with respect to the order induced on $S$ by $\varphi$. We then show that an algebra of $(S, \varphi)$-type degenerates to a quantum toric variety if and only if it has a basis of monomials indexed by $S$. At this stage it is useful to recall the notion of an Algebra with a Straightening Law (see [DCEP82] in the commutative case and [RZ12] in the quantum case). It turns out that, under a mild assumption, this structure provides a natural toric degeneration.

Schubert varieties in the grassmannian enjoy such an ASL structure, both in he classical and quantum case. However, in more general Schubert varieties such a structure no longer exists. The notion of an algebra of $(S, \varphi)$-type is a generalization of the ASL structure which does apply in this more general context, and the aforementioned basis indexed by $S$ plays the role of the standard monomial basis. For more details regarding these two notions see 3.8 . We finish this section by introducing the notion of an $(S,<)$-basis, where $S$ is a semigroup and $<$ is a total order on $S$. It turns out that the existence of such a structure associated to an affine semigroup $S$, with the order obtained by pulling back the lexicographic order of $\mathbb{N}^{t}$ through an embedding $S \hookrightarrow \mathbb{N}^{t}$, is enough to obtain an $(S, \varphi)$-type structure and to prove the existence of a toric degeneration.

Section 4 contains a review of the definitions of quantum flag varieties and their Schubert subvarieties. We then introduce bases for these objects originally defined by Caldero, who showed that they are parametrized by affine semigroups using a variant of Littelmann's string parametrization, and that they have the multiplicative properties needed to produce a toric degeneration. More precisely, we show that these bases are $(S,<)$-bases for an adequate semigroup $S$. We stress that, since Caldero was interested only in classical varieties, he worked over the field $\mathbb{C}(q)$, where $q$ is a transcendental variable, and with an enlarged version of the quantum group $U_{q}(\mathfrak{g})$ (which Janzen calls the adjoint type). Since we are interested in the quantum setting, we work over the ring $\mathbb{Z}\left[v, v^{-1}\right]$ and eventually specialize to an arbitrary field containing a deformation parameter $q$ which is not a root of unity. Furthermore we use the small (simply connected) version of $U_{q}(\mathfrak{g})$.

While technical, we felt that these differences merited a detailed exposition of the arguments, which we present in paragraphs 4.6 to 4.12 . Our proof that Caldero's bases have the desired multiplicative properties is different that the one found in [Calo2]. While he uses multiplicative properties of the dual global basis, we analize the co- 
product of $U_{q}^{-}(\mathfrak{g})$ in terms of the global basis. To do this we are led to study a second basis introduced by Littelman in [Lit98], consisting of certain monomials of divided differences. This monomial basis seems to be better adapted for computations, and has been used to obtain more general degeneration results in the classical case by Fang, Fourier and Littelmann [FFL17b]. However, the global basis, or rather its string parametrization, is crucial in order to prove that one obtains a toric degeneration.

Acknowledgements: The second author would like to thank Xin Fang for an illuminating dicussion on the relation between monomial and canonical bases, and Köln University for its hospitality.

\section{Degeneration of graded connected algebras}

Let $r$ be a positive integer. Throughout this section $A$ denotes a noetherian connected $\mathbb{N}^{r}$-graded $\mathbb{k}$-algebra. Here connected means that the homogeneous component of $A$ of degree $(0, \ldots, 0)$ is isomorphic to $\mathbb{k}$ as a ring, so the ideal generated by all homogeneous elements of non-zero degree is the unique maximal graded ideal of $A$; we denote this ideal by $\mathfrak{m}$. Clearly $A / \mathfrak{m} \cong \mathbb{k}$ as vector spaces, and whenever we consider $\mathbb{k}$ as $A$-bimodule, it will be with this structure.

\section{Graded modules}

2.1. We denote by $\mathbb{Z}^{r}$ Mod $A$ the category of $\mathbb{Z}^{r}$-graded $A$-modules with homogeneous morphisms of degree 0 . We review some general properties of this category; the reader is referred to [NVOo4, chapter 2] for proofs and details.

The category $\mathbb{Z}^{r} \operatorname{Mod} A$ has enough projectives and injectives, so we may speak of the graded projective and injective dimensions of an object $M$, which we denote by $\operatorname{pdim}_{A}^{\mathbb{Z}^{r}} M$ and injdim $\mathbb{Z}_{A}^{r} M$, respectively. We denote by $\mathbb{Z}^{r} \bmod A$ the subcategory of finitely generated $\mathbb{Z}^{r}$-graded $A$-modules. Since $A$ is noetherian $\mathbb{Z}^{r} \bmod A$ is an abelian category with enough projectives.

For every object $M$ of $\mathbb{Z}^{r} \operatorname{Mod} A$ and every $\xi \in \mathbb{Z}^{r}$ we denote by $M_{\xi}$ the homogeneous component of $M$ of degree $\xi$. Also, we denote by $M[\xi]$ the object of $\mathbb{Z}^{r}$ Mod $A$ with the same underlying $A$-module as $M$ and with homogeneous components $M[\xi]_{\zeta}=M_{\zeta+\zeta}$ for all $\zeta \in \mathbb{Z}^{r}$. If $f: N \longrightarrow M$ is a morphism in $\mathbb{Z}^{r} \operatorname{Mod} A$ then the same function defines a morphism $f[\xi]: N[\xi] \longrightarrow M[\xi]$. In this way we get an endofunctor $[\xi]: \mathbb{Z}^{r} \operatorname{Mod} A \longrightarrow \mathbb{Z}^{r} \operatorname{Mod} A$, called the $\xi$-suspension functor; it is an autoequivalence, with inverse $[-\xi]$.

Given $N, M$ objects of $\mathbb{Z}^{r} \operatorname{Mod} A$ we set

$$
\underline{\operatorname{Hom}}_{A}(N, M)=\bigoplus_{\xi \in \mathbb{Z}^{r}} \operatorname{Hom}_{\mathbb{Z}^{r} \operatorname{Mod} A}(N, M[\xi]) .
$$

This is a $\mathbb{Z}^{r}$-graded vector space, with its component of degree $\xi \in \mathbb{Z}^{r}$ equal to the space of homogeneous $A$-linear maps of degree $\xi$ from $N$ to $M$. For every $i \geq 0$ we 
denote by $\underline{\mathrm{Ext}}_{A}^{i}$ the $i$-th right derived functor of $\underline{\mathrm{Hom}}_{A}$. We point out that

$$
\underline{\operatorname{Hom}}_{A}(N[\xi], M) \cong \underline{\operatorname{Hom}}_{A}(N, M[-\xi]) \cong \underline{\operatorname{Hom}}_{A}(N, M)[-\xi],
$$

as $\mathbb{Z}^{r}$-graded vector spaces, and that these isomorphisms induce analogous ones for the corresponding right derived functors.

2.2. Given $\xi=\left(\xi_{1}, \ldots, \xi_{r}\right) \in \mathbb{Z}^{r}$ we set $|\xi|=\xi_{1}+\cdots+\xi_{r}$. Given a $\mathbb{Z}^{r}$-graded vector space $V$, we denote by $|V|$ the $\mathbb{Z}$-graded vector space whose $n$-th homogeneous component is

$$
|V|_{n}=\bigoplus_{|\xi|=n} V_{\tilde{\xi}}
$$

In particular $|A|$ is a connected $\mathbb{N}$-graded algebra. Also, if $M$ is a $\mathbb{Z}^{r}$-graded $A$-module then $|M|$ is a $\mathbb{Z}$-graded $|A|$-module, and this assignation is functorial. Since $A$ is noetherian, [RZ15, Proposition 1.3.7] implies that for every $i \geq 0$ and any pair of $\mathbb{Z}^{r}$ graded modules $N, M$, with $N$ finitely generated, there is an isomorphism of $\mathbb{Z}$-graded modules

$$
\left|\underline{\operatorname{Ext}}_{A}^{i}(N, M)\right| \cong \underline{\operatorname{Ext}}_{|A|}^{i}(|N|,|M|),
$$

natural in both variables.

\section{Homological regularity properties}

In this subsection we discuss some homological properties a connected $\mathbb{N}^{r}$-graded algebra may posses. Most of the material found in this section is standard for connected $\mathbb{N}$-graded algebras.

2.3. Let $M$ be a $\mathbb{Z}^{r}$-graded $A$-module. We say that $\chi(M)$ holds if for each $i \geq 0$ the graded vector space $\operatorname{Ext}_{A}^{i}(\mathbb{k}, M)$ is finite dimensional, and say that the algebra $A$ has property $\chi$ if $\chi(M)$ holds for every finitely generated $\mathbb{Z}^{r}$-graded $A$-module $M$. Property $\chi$ was originally introduced in [AZ94, section 3] and plays a fundamental role in noncommutative algebraic geometry.

2.4. Associated to $A$ and $\mathfrak{m}$ there is a torsion functor

$$
\begin{aligned}
\Gamma_{\mathfrak{m}}: \mathbb{Z}^{r} \operatorname{Mod} A & \longrightarrow \mathbb{Z}^{r} \operatorname{Mod} A \\
M & \longmapsto\left\{x \in M \mid \mathfrak{m}^{n} x=0 \text { for } n \gg 0\right\},
\end{aligned}
$$

which acts on morphisms by restriction and correstriction. The torsion functor is left exact, and for each $i \geq 0$ its $i$-th right derived functor is denoted by $H_{\mathfrak{m}}^{i}$ and called the $i$-th local cohomology functor of $A$.

There exists a natural isomorphism

$$
\Gamma_{\mathfrak{m}} \cong \underset{n}{\lim } \underline{\operatorname{Hom}}_{A}\left(A / \mathfrak{m}^{n},-\right)
$$


which by standard homological algebra extends to natural isomorphisms

$$
H_{\mathfrak{m}}^{i} \cong \underset{n}{\lim } \underline{\operatorname{Ext}}_{A}^{i}\left(A / \mathfrak{m}^{n},-\right)
$$

for all $i \geq 1$. The proof of this fact is completely analogous to the one found in [BS98, Theorem 1.3.8] for commutative ungraded algebras.

We denote by $A^{\circ}$ the opposite algebra of $A$, which is also a connected $\mathbb{N}^{r}$-graded algebra, and by $\mathfrak{m}^{\circ}$ its maximal graded ideal. We write $\Gamma_{\mathfrak{m}^{\circ}}$ and $H_{\mathfrak{m}^{\circ}}^{i}$ for the corresponding torsion and local cohomology functors, respectively.

2.5. Given an object $M$ of $\mathbb{Z}^{r} \operatorname{Mod} A$, its depth and local dimension are defined as

$$
\begin{aligned}
\operatorname{depth}_{\mathfrak{m}} M & =\inf \left\{i \in \mathbb{N} \mid \underline{\text { Ext }}_{A}^{i}(\mathbb{k}, M) \neq 0\right\}, \\
\lim _{\mathfrak{m}} M & =\sup \left\{i \in \mathbb{N} \mid H_{\mathfrak{m}}^{i}(M) \neq 0\right\},
\end{aligned}
$$

respectively. The local cohomological dimension of $A$, denoted by $\operatorname{lcd}_{\mathfrak{m}} A$, is the supremum of the $\operatorname{ldim}_{\mathfrak{m}} M$ with $M$ finitely generated.

2.6. The following definition is taken from [RZ15, Definition 2.1.1]. It is an $\mathbb{N}^{r}$-graded analogue of the definition of the AS-Cohen-Macaulay, AS-Gorenstein and AS-regular properties for connected $\mathbb{N}$-graded algebras found in the literature, see for example the introduction to [JZoo].

Definition. Let $A$ be a connected noetherian $\mathbb{N}^{r}$-graded algebra.

1. A is called AS-Cohen-Macaulay if there exists $n \in \mathbb{N}$ such that $H_{\mathfrak{m}}^{i}(A)=0$ and $H_{\mathfrak{m}^{\circ}}^{i}(A)=0$ for all $i \neq n$.

2. $A$ is called left AS-Gorenstein if it has finite graded injective dimension $n$ and there exists $\ell \in \mathbb{Z}^{r}$, called the Gorenstein shift of $A$, such that

$$
\underline{\operatorname{Ext}}_{A}^{i}(\mathbb{k}, A) \cong \begin{cases}\mathbb{k}[\ell] & \text { for } i=n, \\ 0 & \text { for } i \neq n,\end{cases}
$$

as $\mathbb{Z}^{r}$-graded $A^{\circ}$-modules. We say $A$ is right $A S$-Gorenstein if $A^{\circ}$ is left AS-Gorenstein. Finally $A$ is AS-Gorenstein if $A$ and $A^{\circ}$ are left AS-Gorenstein, with the same injective dimensions and Gorenstein shifts.

3. A is called AS-regular if it is AS-Gorenstein, and its left and right graded global dimensions are finite and equal.

2.7. The properties discussed in paragraphs 2.3 to 2.6 are defined in terms of the category of $\mathbb{Z}^{r}$-graded $A$-modules. In 2.2 we defined the algebra $|A|$, which is equal to $A$ as algebra but is endowed with a connected $\mathbb{N}$-grading induced by the grading on $A$ and the group morphism $|\cdot|$. The maximal graded ideals of $A$ and $|A|$ coincide as vector spaces, so we may ask whether the fact that $A$ has property $\chi$, or finite local dimension, or the AS-Cohen-Macaulay property, etc., implies that $|A|$ has the corresponding property. 
Let us say that a property $P$ does not depend on the grading of $A$ if the following holds: for every connected $\mathbb{N}^{t}$-graded algebra $B$ with maximal ideal $\mathfrak{n}$, which is isomorphic to $A$ as algebra through an isomorphism that sends $\mathfrak{n}$ to $\mathfrak{m}, A$ has property $P$ if and only if $B$ has property $P$. As shown in [RZ15, Corollary 1.3.9], the local dimension of $A$ does not depend on the grading of $A$. An analogous result is proved in [RZ15, Remark 2.1.7] for the properties defined in 2.6. However, the situation is more delicate for property $\chi$. The following lemma shows that property $\chi$ is independent of the grading of $A$ under the hypothesis that $A$ has finite local dimension; we do not know whether this hypothesis can be eliminated.

Lemma. Suppose $\operatorname{lcd}_{\mathfrak{m}} A<\infty$. Then the algebra $A$ has property $\chi$ if and only if $\chi(A)$ holds.

Proof. If $A$ has property $\chi$ then clearly $\chi(A)$ holds. To prove the opposite implication, assume $\chi(A)$ holds. Recall from 2.2 that for every $\mathbb{Z}^{r}$-graded $A$-module $M$ and for every $i \geq 0$ there exists a graded vector space isomorphism

$$
\left|\underline{\operatorname{Ext}}_{A}^{i}(\mathbb{k}, M)\right| \cong \underline{\operatorname{Ext}}_{|A|}^{i}(|\mathbb{k}|,|M|),
$$

so $\chi(M)$ holds if and only if $\chi(|M|)$ holds; in view of this, the hypothesis implies $\chi(|A|)$ holds.

Since $\operatorname{ldim}_{|\mathfrak{m}|}|A|=\operatorname{dim}_{\mathfrak{m}} A<\infty$, we may apply [RZ15, Proposition 2.2.6] and conclude that $|A|$ has property $\chi$. From this it follows that $\chi(|M|)$ holds for every $\mathbb{Z}^{r}$-graded $A$-module $M$, and hence so does $\chi(M)$.

2.8. We finish this subsection with a technical result on the relation between property $\chi$ and local cohomology.

Lemma. For every $n \in \mathbb{N}$, let $A_{\geq n}$ be the ideal generated by all homogeneous elements of degree $\xi$ with $|\xi| \geq n$. Let $M$ be a finitely generated $\mathbb{Z}^{r}$-graded A-module such that $\chi(M)$ holds. Then for every $i \geq 0$ and every $t \in \mathbb{Z}$ there exists $n_{0} \in \mathbb{Z}$ such that

$$
\underline{\operatorname{Ext}}_{A}^{i}\left(A / A_{\geq n}, M\right)_{\xi} \cong H_{\mathfrak{m}}^{i}(M)_{\xi}
$$

for all $n \geq n_{0}$ and all $\xi \in \mathbb{Z}^{r}$ such that $|\xi| \geq t$.

Proof. Since $\mathfrak{m}$ is finitely generated, say by elements $x_{1}, \ldots, x_{r}$ with degrees $\xi_{1}, \ldots, \xi_{r}$ such that $\left|\xi_{i}\right| \geq 1$, clearly $\mathfrak{m}^{n} \subset A_{\geq n}$. Setting $l=\max \left\{\left|\xi_{i}\right|: 1 \leq i \leq r\right\}$ we obtain $A_{\geq \ln } \subset \mathfrak{m}^{n}$. Knowing this, the proof of [BS98, Proposition 3.1.1] easily adapts to show that for every $i \geq 0$ there exist natural isomorphisms

$$
\underset{n}{\lim } \operatorname{Ext}_{A}^{i}\left(A / A_{\geq n},-\right) \cong \underset{n}{\lim _{n}} \underline{\operatorname{Ext}}_{A}^{i}\left(A / \mathfrak{m}^{n},-\right) \cong H_{\mathfrak{m}}^{i} .
$$

The statement of the lemma will follow if we show that for all $\xi$ as in the statement, the homogeneous component of degree $\xi$ of the natural map

$$
\pi^{n}: \underline{\operatorname{Ext}}_{A}^{i}\left(A / A_{\geq n}, M\right) \longrightarrow H_{\mathfrak{m}}^{i}(M)
$$

is an isomorphism for $n \gg 0$. 
Fixing $t$ as in the statement, [AZ94, Proposition 3.5 (1)] implies that the natural map $\pi_{d}^{n}:$ Ext $_{|A|}^{i}\left(|A| /\left|A_{\geq n}\right|,|M|\right)_{d} \longrightarrow H_{|\mathfrak{m}|}^{i}(|M|)_{d}$ is an isomorphism for all $d \geq t$ if $n$ is large enough. Now by [RZ15, Propositions 1.3.7 and 1.3.8] there exist isomorphisms

$$
\begin{aligned}
\underline{\operatorname{Ext}}_{|A|}^{i}\left(|A| /\left|A_{\geq n}\right|,|M|\right)_{d} & \cong \bigoplus_{|\xi|=d}{\underline{\operatorname{Ext}_{A}^{i}}}_{A}\left(A / A_{\geq n}, M\right)_{\xi} \\
H_{|\mathfrak{m}|}^{i}(|M|)_{d} & \cong \bigoplus_{|\xi|=d} H_{\mathfrak{m}}^{i}(M)_{\xi}
\end{aligned}
$$

Since the assignation $M \mapsto|M|$ is functorial, we also get that $\pi_{d}^{n}=\bigoplus_{|\xi|=d} \pi_{\xi}^{n}$. Thus for all $\xi$ such that $|\xi| \geq t$, the map $\pi_{\xi}^{n}$ is an isomorphism if $n$ is large enough.

\section{Transfer of regularity properties by degeneration}

In this subsection we prove that if $A$ has a filtration compatible with its grading, and the associated graded algebra has property $\chi$, then the regularity properties discussed in the previous subsection transfer from $\operatorname{gr} A$ to $A$. All undefined terms regarding filtrations can be found in [NVO79, chapter I].

2.9. Recall that $A$ denotes a noetherian $\mathbb{N}^{r}$-graded algebra. The general setup for the subsection is as follows: we assume that $A$ has a connected filtration, that is an exhaustive filtration $\mathcal{F}=\left\{F_{n} A\right\}_{n \geq 0}$, with $\mathbb{k}=F_{0} A \subset F_{1} A \subset \cdots \subset F_{n} A \subset \cdots \bigcup_{n \geq 0} F_{n} A=A$, such that each layer $F_{n} A$ is a finite dimensional graded vector space, and $\bar{F}_{n} A \cdot F_{m} A \subset$ $F_{n+m} A$ for all $n, m \in \mathbb{N}$. For each $\xi \in \mathbb{N}^{r}$ the homogeneous component $A_{\xi}$ has an induced filtration $\left\{F_{n} A_{\xi}\right\}_{n \geq 0}$, where $F_{n} A_{\xi}=F_{n} A \cap A_{\xi}$. Since $A_{\xi}$ is finite dimensional this filtration is finite, so the associated graded $\operatorname{ring} \operatorname{gr} A$ is a connected and locally finite $\mathbb{N}^{r+1}$-graded algebra.

Given any $\mathbb{Z}^{r}$-graded $A$-module $M$ with a filtration whose layers are $\mathbb{Z}^{r}$-graded subspaces, we can construct the $\mathbb{Z}^{r+1}$-graded gr $A$-module gr $M$. If $M$ is any $\mathbb{Z}^{r}$-graded $A$-module then it can be endowed with such a filtration as follows: fix a graded subspace $N \subset M$ that generates $M$ over $A$, and for each $n \geq 0$ set $F_{n} M=\left(F_{n} A\right) N$. Any such filtration is called standard, and is an exhaustive and discrete filtration by graded subspaces. If $M$ is finitely generated and $N$ is finite dimensional then the layers of this filtration are also finite dimensional.

2.10. The main tool used to transfer homological information from gr $A$ to $A$ is a spectral sequence that we associate to any pair of $\mathbb{Z}^{r}$-graded $A$-modules $N, M$, which converges to $\operatorname{Ext}_{A}^{i}(N, M)$ and whose first page consists of the homogeneous components of Ext $\mathrm{gr}_{A}^{i}(\mathrm{gr} N, \mathrm{gr} M)$. The proof is straightforward, but relies on several graded analogues of classical constructions for filtered rings. These constructions can be found in [NVO79, Chapter I] and [MRo1, Section 7.6], and the proofs found in the references easily adapt to the graded context, so we use them without further comment.

In order to keep track of the extra component in the grading when passing to associated graded objects, we make a slight abuse of notation: given a $\mathbb{Z}^{r+1}$-graded vector space $V$, we denote by $V_{(\xi, p)}$ its homogeneous component of degree $\left(\xi_{1}, \ldots, \xi_{r}, p\right)$. 
Proposition. Let $A$ be an $\mathbb{N}^{r}$-graded algebra with a connected filtration, and assume $\operatorname{gr} A$ is noetherian. Let $M, N$ be filtered $\mathbb{Z}^{r}$-graded $A$-modules, with $N$ finitely generated, and suppose that the filtration on $N$ is standard and the filtration on $M$ is discrete. Then for every $\xi \in \mathbb{Z}^{r}$ there exists a convergent spectral sequence

$$
E(N, M)_{\xi}: E_{p, q}^{1}={\underline{\operatorname{Ext}_{g r} A}}^{-p-q}(\operatorname{gr} N, \operatorname{gr} M)_{(\xi, p)} \Rightarrow{\underline{\operatorname{Ext}_{A}^{-p-q}}}^{-1}(N, M)_{\xi} \quad p, q \in \mathbb{Z},
$$

such that the filtration of the vector spaces on the right hand side is finite.

Proof. By the $\mathbb{Z}^{r}$-graded version of [MRo1, Theorem 6.17], there exists a projective resolution $P^{\bullet} \longrightarrow N$ by filtered projective $\mathbb{Z}^{r}$-graded $A$-modules with filtered differentials, such that the associated graded complex gr $P^{\bullet} \longrightarrow \operatorname{gr} N$ is a $\mathbb{Z}^{r+1}$-graded projective resolution of the gr $A$-module gr $N$. Using the filtration for the Hom spaces defined in [NVO79, section I.2], the complex $\underline{\operatorname{Hom}}_{A}\left(P^{\bullet}, M\right)$ is a graded complex with a filtration by graded subcomplexes, whose differentials are filtered maps.

If we fix $\xi \in \mathbb{Z}^{r}$, the homogeneous component $\underline{\operatorname{Hom}}_{A}\left(P^{\bullet}, M\right)_{\xi}$ is a complex of filtered finite dimensional vector spaces. By [Weig4, 5.5.1.2] there exists a spectral sequence with page one equal to

$$
E_{p, q}^{1}=H_{p+q}\left(\frac{F_{p} \underline{\operatorname{Hom}}_{A}\left(P^{\bullet}, M\right)_{\xi}}{F_{p-1} \underline{\operatorname{Hom}}_{A}\left(P^{\bullet}, M\right)_{\xi}}\right) \quad p, q \in \mathbb{Z}
$$

that converges to

$$
H_{p+q}\left(\underline{\operatorname{Hom}}_{A}\left(P^{\bullet}, M\right)_{\xi}\right) \cong{\underline{\operatorname{Ext}_{A}}}_{A}^{-p-q}(N, M)_{\xi} .
$$

This last space is finite dimensional, and hence the filtration on it is finite. Thus we only need to prove that for each $p, q \in \mathbb{Z}$ there exists an isomorphism

$$
H_{p+q}\left(\frac{F_{p} \underline{\operatorname{Hom}}_{A}\left(P^{\bullet}, M\right)_{\xi}}{F_{p-1} \underline{\operatorname{Hom}}_{A}\left(P^{\bullet}, M\right)_{\xi}}\right) \cong \underline{\operatorname{Ext}}_{\mathrm{gr} A}^{-p-q}(\operatorname{gr} N, \operatorname{gr} M)_{(\xi, p)} .
$$

By [NVO79, Lemma 6.4], there exists an isomorphism of complexes

$$
\varphi\left(P^{\bullet}, M\right): \operatorname{gr}\left(\underline{\operatorname{Hom}}_{A}\left(P^{\bullet}, M\right)\right) \longrightarrow \underline{\operatorname{Hom}}_{\operatorname{gr} A}\left(\operatorname{gr} P^{\bullet}, \operatorname{gr} M\right),
$$

which is defined explicitly in the reference. Direct inspection shows that the map $\varphi\left(P^{\bullet}, M\right)$ is homogeneous, so looking at its component of degree $\xi$ we obtain an isomorphism

$$
\frac{F_{p} \underline{\operatorname{Hom}}_{A}\left(P^{\bullet}, M\right)_{\xi}}{F_{p-1} \underline{\operatorname{Hom}}_{A}\left(P^{\bullet}, M\right)_{\xi}} \cong \underline{\operatorname{Hom}}_{\operatorname{gr} A}\left(\operatorname{gr} P^{\bullet}, \operatorname{gr} M\right)_{(\xi, p)} .
$$

Since gr $P^{\bullet}$ is a $\mathbb{Z}^{r+1}$-graded projective resolution of $\operatorname{gr} N$, we obtain the desired isomorphism by applying $H_{p+q}$ to both sides of the isomorphism.

The following Corollary is an immediate consequence of the previous Proposition. 
Corollary. Let $A$ be an $\mathbb{N}^{r}$-graded algebra with a connected filtration, and assume $\operatorname{gr} A$ is noetherian. Let $M, N$ be filtered $\mathbb{Z}^{r}$-graded $A$-modules, with $N$ finitely generated, and suppose that the filtration on $N$ is standard and the filtration on $M$ is discrete. Then the following hold.

(a) For each $i \geq 0$ and each $\xi \in \mathbb{Z}^{r}$

$$
\operatorname{dim}_{\mathbb{k}} \underline{\operatorname{Ext}}_{A}^{i}(N, M)_{\xi} \leq \sum_{p=-\infty}^{\infty} \operatorname{dim}_{\mathbb{k}} \underline{\operatorname{Ext}}_{\mathrm{gr} A}^{i}(\operatorname{gr} N, \operatorname{gr} M)_{(\xi, p)}
$$

(b) $\operatorname{pdim}_{A}^{\mathbb{Z}^{r}} N \leq \operatorname{pdim}_{\mathrm{gr} A}^{\mathbb{Z}^{r+1}} \operatorname{gr} N$ and injdim $\mathbb{Z}_{A}^{\mathbb{Z}^{r}} M \leq \operatorname{injdim} \operatorname{gr}_{A}^{\mathbb{Z}^{r+1}} \operatorname{gr} M$.

(c) If $\chi(\operatorname{gr} M)$ holds then $\chi(M)$ holds.

2.11. We now prove a result that relates the local cohomology of a $\mathbb{Z}^{r}$-graded $A$ module $M$ with that of its associated graded module gr $M$. We will do this by combining Proposition 2.10 with Lemma 2.8 and the formalism of the change of grading functors introduced in [RZ12, Section 1.3]. We recall the relevant details. Given a group morphism $\varphi: \mathbb{Z}^{r} \longrightarrow \mathbb{Z}^{t}$, with $t \in \mathbb{N}$, there exists a functor $\varphi_{!}: \mathbb{Z}^{r} \operatorname{Mod} \mathbb{k} \longrightarrow \mathbb{Z}^{t} \operatorname{Mod} \mathbb{k}$ that sends a $\mathbb{Z}^{r}$-graded vector space $V$ to the $\mathbb{Z}^{t}$-graded vector space $\varphi_{!}(V)$, whose homogeneous component of degree $\zeta \in \mathbb{Z}^{t}$ is

$$
V_{\zeta}=\bigoplus_{\varphi(\xi)=\zeta} V_{\xi}
$$

Notice that $\varphi_{\text {! }}$ does not change the underlying vector space of its argument, only its grading. If $R$ is a $\mathbb{Z}^{r}$-graded algebra then $\varphi_{!}(R)$ is a $\mathbb{Z}^{t}$-graded algebra, and if $M$ is a $\mathbb{Z}^{r}$ graded $R$-module then $\varphi_{!}(M)$ is a $\mathbb{Z}^{t}$-graded $\varphi_{!}(R)$-module with the same underlying $R$-module structure as $M$.

Corollary. Let $A$ be an $\mathbb{N}^{r}$-graded algebra with a connected filtration, and assume gr $A$ is noetherian. Let $M$ be a filtered $\mathbb{Z}^{r}$-graded A-module with a discrete filtration, and assume $\chi(\operatorname{gr} M)$ holds. Then for each $i \geq 0$ and each $\xi \in \mathbb{Z}^{r}$

$$
\operatorname{dim}_{\mathbb{k}} H_{\mathfrak{m}}^{i}(M)_{\xi} \leq \sum_{p=-\infty}^{\infty} \operatorname{dim}_{\mathbb{k}} H_{\mathrm{gr} \mathrm{m}}^{i}(\operatorname{gr} M)_{(\xi, p)} .
$$

Proof. Let $\pi: \mathbb{Z}^{r+1} \longrightarrow \mathbb{Z}^{r}$ be the projection to the first $r$-coordinates, and set $B=$ $\pi_{!}(\operatorname{gr} A)$, so for every $\xi \in \mathbb{Z}^{r}$

$$
B_{\xi}=\bigoplus_{p \in \mathbb{Z}} \operatorname{gr} A_{(\xi, p)}=\operatorname{gr}\left(A_{\tilde{\zeta}}\right)
$$

Thus $B$ is a connected $\mathbb{N}^{r}$-graded algebra. Set $\mathfrak{n}=\pi_{!}(\operatorname{gr} \mathfrak{m})$, which is the maximal graded ideal of $B$, and set $\tilde{M}=\pi_{!}(\operatorname{gr} M)$. 
By [RZ15, Proposition 1.3.7] $\chi($ gr $M)$ implies $\chi(\tilde{M})$ and by item (c) of Corollary 2.10 it also implies $\chi(M)$, so we may apply Lemma 2.8 to $M$ and $\tilde{M}$, and deduce that for given $\xi \in \mathbb{Z}^{r}$ and $i \geq 0$ the natural maps

$$
\begin{aligned}
\underline{\operatorname{Ext}}_{A}^{i}\left(A / A_{\geq n}, M\right)_{\xi} & \longrightarrow H_{\mathfrak{m}}^{i}(M)_{\xi} \\
\underline{\operatorname{Ext}}_{B}^{i}\left(B / B_{\geq n}, \tilde{M}\right)_{\xi} & \longrightarrow H_{\mathfrak{n}}^{i}(\tilde{M})_{\xi}
\end{aligned}
$$

are isomorphisms for $n \gg 0$.

Combining this with [RZ15, Propositions 1.3 .7 and 1.3.8], we obtain a chain of isomorphisms

$$
\begin{aligned}
\bigoplus_{p \in \mathbb{Z}} \underline{\operatorname{Ext}}_{\mathrm{gr} A}^{i} & \left(\frac{\operatorname{gr} A}{\operatorname{gr}\left(A_{\geq n}\right)}, \operatorname{gr} M\right)_{(\xi, p)} \\
& \cong \underline{\operatorname{Ext}}_{B}^{i}(B / B \geq n, \tilde{M})_{\xi} \cong H_{\mathfrak{n}}^{i}(\tilde{M})_{\xi} \cong \bigoplus_{p \in \mathbb{Z}} H_{\mathrm{gr} \mathfrak{m}}^{i}(\operatorname{gr} M)_{(\xi, p)}
\end{aligned}
$$

By definition $\operatorname{gr}\left(A / A_{\geq n}\right) \cong \operatorname{gr} A / \operatorname{gr}\left(A_{\geq n}\right)$, so applying item (a) of Corollary [2.10 and taking $n \gg 0$ we obtain

$$
\begin{aligned}
\operatorname{dim}_{\mathbb{k}} H_{\mathfrak{m}}^{i}(M)_{\xi} & =\operatorname{dim}_{\mathbb{k}} \underline{\operatorname{Ext}}_{A}^{i}\left(A / A_{\geq n}, M\right)_{\xi} \\
& \leq \sum_{p=-\infty}^{\infty} \operatorname{dim}_{\mathbb{k}} \underline{\operatorname{Ext}}_{\mathrm{gr} A}^{i}\left(\operatorname{gr} A / \operatorname{gr}\left(A_{\geq n}\right), M\right)_{(\xi, p)} \\
& =\sum_{p=-\infty}^{\infty} \operatorname{dim}_{\mathbb{k}} H_{\mathrm{gr} \mathrm{m}}^{i}(\operatorname{gr} M)_{(\xi, p)} .
\end{aligned}
$$

2.12. We are now ready to prove the main result of this section.

Theorem. Suppose A is a connected $\mathbb{N}^{r}$-graded algebra endowed with a connected filtration, and that $\operatorname{gr} A$ is noetherian and has property $\chi$. Then the following hold.

(a) A has property $\chi$.

(b) $\operatorname{lcd}_{\mathfrak{m}} A \leq \operatorname{lcd}_{\operatorname{grm}} \operatorname{gr} A$.

(c) If $\mathrm{gr} A$ is AS-Cohen-Macaulay, AS-Gorenstein or AS-Regular, so is A.

Proof. The hypothesis that $\operatorname{gr} A$ is noetherian implies that $A$ is noetherian [MRo1, 1.6.9]. If $M$ is any finitely generated $\mathbb{Z}^{r}$-graded $A$-module, we may filter it using the procedure described in 2.9. Since $\chi($ gr $M)$ holds by hypothesis, item (c) of Corollary 2.10 implies $\chi(M)$ holds, which proves that $A$ has property $\chi$.

Item 2 follows from Corollary 2.11, as does the fact that if $\operatorname{gr} A$ is AS-CohenMacaulay so is $A$. If $\mathrm{gr} A$ is AS-Gorenstein of injective dimension $n$ and Gorenstein shift $(\xi, p) \in \mathbb{Z}^{r+1}$, the algebra $A$ has injective dimension at most $n$ by item (b) of Corollary 2.10, Also, for each $\zeta \in \mathbb{Z}^{r}$ the spectral sequence $E(\mathbb{k}, A)_{\zeta}$ of Proposition 
2.10 degenerates at page 1: it is zero if $\zeta \neq-\xi$, while for $\zeta=-\xi$ there is a single, one dimensional non-zero entry in the diagonal $-p-q=n$. Hence we obtain vector space isomorphisms

$$
\underline{\operatorname{Ext}}_{A}^{i}(\mathbb{k}, A)_{\zeta} \cong \begin{cases}\mathbb{k} & \text { if } \zeta=-\xi \text { and } i=n \\ 0 & \text { otherwise. }\end{cases}
$$

Thus $\underline{\operatorname{Ext}}_{A}^{i}(\mathbb{k}, A) \cong \mathbb{k}[\xi]$ as $\mathbb{Z}^{r}$-graded vector spaces. Any such isomorphism is also $A^{\circ}$-linear, so $A$ is left AS-Gorenstein of injective dimension $n$ and Gorenstein shift $\xi$. The same proof applies to show that $A$ is right AS-Gorenstein with the same injective dimension and Gorenstein shift, so $A$ is AS-Gorenstein.

Finally, assume that $\operatorname{gr} A$ is AS-regular. Then $A$ is AS-Gorenstein, and item (b) of Corollary 2.10 implies that it has finite left and right global dimensions. These dimensions are equal to the left and right projective dimensions of $\mathbb{k}$ as $A$-module [RZ15, Lemma 2.1.5], which in turn equal the left and right injective dimensions of $A$ and hence coincide, so $A$ is AS-regular.

\section{Quantum affine toric degenerations}

In this section we recall the homological properties of quantum affine toric varieties proved in [RZ15], and give necessary and sufficient conditions for a connected $\mathbb{N}^{r}$ graded algebra $A$ to have a connected filtration such that the associated graded ring is a quantum positive affine toric variety.

\section{Quantum affine toric varieties}

3.1. Recall that an affine semigroup is a finitely generated monoid isomorphic to a subsemigroup of $\mathbb{Z}^{r}$ for some $r \in \mathbb{N}$. An embedding of $S$ is an injective semigroup morphism $i: S \hookrightarrow \mathbb{Z}^{r}$ for some $r \in \mathbb{N}$. By definition, every affine semigroup $S$ is commutative and cancellative, so it has a group of fractions, which we denote by $G(S)$, and the natural map from $S$ to $G(S)$ is injective. The group $G(S)$ is a finitely generated and torsion-free commutative group, so there exists a natural number $r$ such that $G(S) \cong \mathbb{Z}^{r}$ as groups; we refer to $r$ as the rank of $S$ and denote it $r k S$. We say that $i$ is a full embedding if the image of $S$ generates $\mathbb{Z}^{r}$ as a group, in which case $r=\operatorname{rk} S$. Fixing an isomorphism $G(S) \cong \mathbb{Z}^{\mathrm{rk}(S)}$ we obtain a full embedding of $S$ in an obvious way.

3.2. Let $S$ be an affine semigroup. Fixing an embedding $i: S \hookrightarrow \mathbb{Z}^{r}$ we identify $S$ with its image and see $\mathbb{Z}^{r}$ as a subgroup of $\mathbb{R}^{r}$ in the obvious way. The rational cone generated by $S$ in $\mathbb{R}^{r}$ is the set

$$
\mathbb{R}_{+} S=\left\{\sum_{i=1}^{n} r_{i} s_{i} \mid r_{i} \in \mathbb{R}_{\geq 0}, s_{i} \in S, n \in \mathbb{N}\right\}
$$


The relative interior of $S$ is defined as relint $S=S \cap \mathbb{R}_{+} S^{\circ}$, where $\mathbb{R}_{+} S^{\circ}$ is the topological interior of $\mathbb{R}_{+} S$ as a subset of the vector space $\mathbb{R} S$ endowed with the subspace topology induced from $\mathbb{R}^{r}$. The relative interior is intrinsic to $S$ and does not depend on the chosen embedding [BGog, Remark 2.6].

An affine semigroup $S$ of rank $r$ is called normal if it verifies the following property: given $z \in G(S)$, if there exists $m \in \mathbb{N}^{*}$ such that $m z \in S$, then $z \in S$. If we identify $S$ with a subset of $\mathbb{Z}^{r}$ through a full embedding and consider the real cone $\mathbb{R}_{+} S$ of $S$ inside $\mathbb{R}^{r}$ in the obvious way, then Gordan's lemma [BH93, Proposition 6.1.2] states that $S$ is normal if and only if $S=\mathbb{Z}^{r} \cap \mathbb{R}_{+} S$.

Example. We now introduce an example that will recur through the rest of this section. Recall that a lattice is a poset $\mathcal{L}$ such that any two elements $x, y$ have an infimum, called the meet of $x$ and $y$ and denoted $x \wedge y$, and a supremum, called the join of $x$ and $y$ and denoted $x \vee y$. For example, given $u \in \mathbb{N}$ the poset $\mathbb{N}^{u}$ with the product order is a lattice, with join (resp. meet) given by taking the maximum (resp. minimum) at each coordinate. A lattice is said to be finite if its underlying set is finite, and distributive if the binary operation $\wedge$ is distributive over $\vee$, and vice versa. Clearly $\mathbb{N}^{u}$ is a distributive lattice, although of course not a finite one. Given $u, v \in \mathbb{N}$ with $u \leq v$, the set $\Pi_{u, v}$ consisting of all $\left(a_{1}, \ldots a_{u}\right) \in \mathbb{N}^{u}$ such that $1 \leq a_{1}<\cdots<a_{u} \leq v$ is a finite distributive sublattice of $\mathbb{N}^{u}$.

An element $z$ of a distributive lattice is said to be join-irreducible if it is not the minimum, and whenever $z=x \vee y$ then $z=x$ or $z=y$. Let $\mathcal{L}$ be a distributive lattice, and let $J(\mathcal{L})=\left\{x_{1}, \ldots, x_{r}\right\}$ be the set of its join irreducible elements, enumerated so that $x_{i}<x_{j}$ implies $i<j$. Following [RZ15, subsection 3.3] set $\operatorname{str}(\mathcal{L})$ to be the subset of $\mathbb{N}^{r+1}$ consisting of tuples $\left(a_{0}, a_{1}, \ldots, a_{r}\right)$ such that $a_{0} \geq a_{i}$ for all $i=1, \ldots, r$, and $a_{i} \geq a_{j}$ whenever $x_{i}<x_{j}$ as elements of $\mathcal{L}$. It turns out that $\operatorname{str}(\mathcal{L})$ is a normal affine semigroup, which we will call the affine semigroup associate to $\mathcal{L}$. Later on we will give an equivalent definition of $S(\mathcal{L})$ in terms of the lattice, which will justify its name. Below we give an example with $\mathcal{L}=\Pi_{2,4}$.

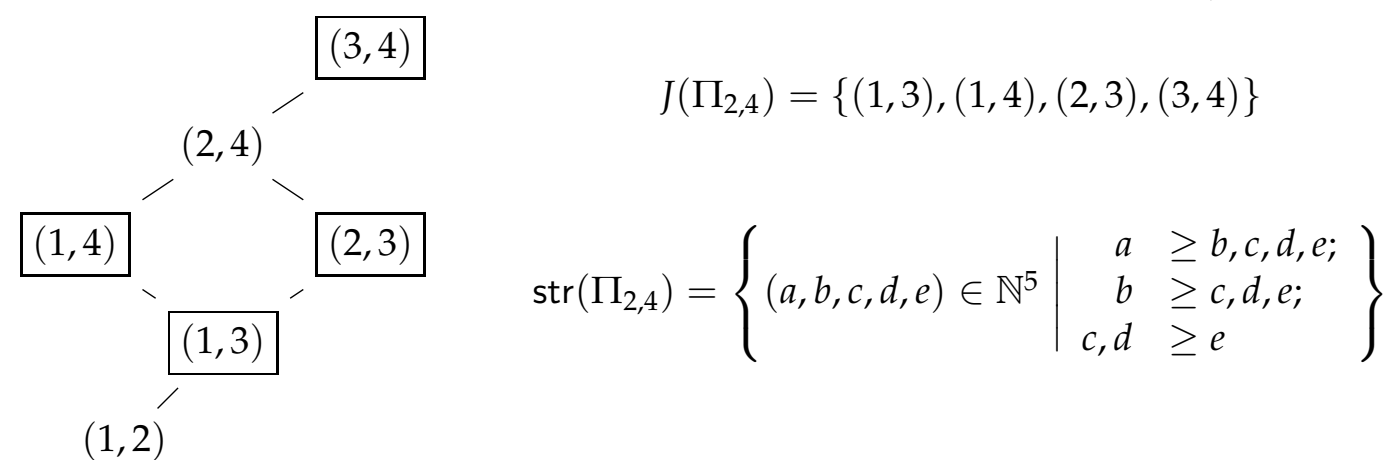

The lattice $\Pi_{2,4}$ and its affine semigroup. Framed elements are join-irreducibles.

3.3. Let $S$ be an affine semigroup. An element $s \in S$ is called irreducible if whenever $S=x+y$ with $x, y \in S$, then either $x$ or $y$ is invertible. On the other hand $S$ is called positive if its only invertible element is 0 . If $S$ is positive then the set of its irreducible elements is finite and generates $S$; for this reason it is called the Hilbert basis of $S$. The 
fact that $S$ is positive also implies that there exists a full embedding $S \hookrightarrow \mathbb{Z}^{r}$ such that the image of $S$ is contained in $\mathbb{N}^{r}$. It follows that a semigroup is positive if and only if there exists $r \geq 0$ such that $S$ is isomorphic to a finitely generated subsemigroup of $\mathbb{N}^{r}$. Proofs of these results can be found in [BGo9, pp. 54-56].

3.4. Let $S$ be a positive affine semigroup and let $\left\{s_{1}, \ldots, s_{n}\right\}$ be its Hilbert Basis. We denote by $\pi: \mathbb{N}^{n} \longrightarrow S$ the semigroup morphism defined by the assignation $e_{i} \mapsto s_{i}$ for each $1 \leq i \leq n$, where $e_{i}$ is the $i$-th element in the canonical basis of $\mathbb{N}^{n}$. This map determines an equivalence relation in $\mathbb{N}^{n}$ where $p \sim p^{\prime}$ if and only if $\pi(p)=\pi\left(p^{\prime}\right)$; we denote this relation by $L(\pi)$. Clearly $L(\pi)$ is compatible with the additive structure of $\mathbb{N}^{n}$, and hence the quotient $\mathbb{N}^{n} / L(\pi)$ is a commutative monoid with the operation induced by addition in $\mathbb{N}^{n}$, and there is an isomorphism of monoids $\mathbb{N}^{n} / L(\pi) \cong S$.

In general, an equivalence relation on $\mathbb{N}^{n}$ closed under addition is called a congruence. If $\rho$ is a subset of $\mathbb{N}^{n} \times \mathbb{N}^{n}$ then the congruence generated by $\rho$ is the smallest congruence containing the set $\rho$. Redei's theorem [RGS99. Theorem 5.12] states that every congruence in $\mathbb{N}^{n}$ is finitely generated, i.e. there exists a finite set $\rho \subset \mathbb{N}^{n} \times \mathbb{N}^{n}$ that generates it; in particular there exists a finite set $P=\left\{\left(p_{1}, p_{1}^{\prime}\right), \ldots,\left(p_{m}, p_{m}^{\prime}\right)\right\} \subset L(\pi)$ which generates $L(\pi)$. A presentation of $S$ will be for us a pair $(\pi, P)$, where $\pi: \mathbb{N}^{n} \longrightarrow$ $S$ is the map described above and $P$ is a finite generating set of the congruence $L(\pi)$. By the previous discussion every positive affine semigroup has a presentation.

Example. Let $\mathcal{L}$ be a finite distributive lattice, and let $F(\mathcal{L})$ be the free commutative monoid on $\mathcal{L}$, which is clearly isomorphic to $\mathbb{N}|\mathcal{L}|$. We will give a presentation of the group $\operatorname{str}(\mathcal{L})$ introduced in 3.2 as a quotient of $F(\mathcal{L})$.

As before, we denote by $\left\{x_{1}, \ldots, x_{r}\right\}$ the set of join-irreducible elements of $\mathcal{L}$. We denote by [l] the image of an element $l \in \mathcal{L}$ in $F(\mathcal{L})$ and define a map $\pi: F(\mathcal{L}) \longrightarrow \operatorname{str}(\mathcal{L})$ given by $\pi([l])=e_{0}+\sum_{x_{i} \leq l} e_{i}$. As shown in [RZ15. Proposition 3.3.3] this map is surjective. Furthermore $L(\pi)$ is the equivalence relation generated by the set $\left\{\left([l]+\left[l^{\prime}\right],\left[l \vee l^{\prime}\right]+\left[l \wedge l^{\prime}\right]\right) \mid\right.$ $\left.l, l^{\prime} \in \mathcal{L}\right\}$. Notice also that the Hilbert basis of $\operatorname{str}(\mathcal{L})$ is precisely the image of $\mathcal{L}$, and the map $\pi$ restricted to $\mathcal{L}$ is a lattice morphism. Thus every distributive lattice $\mathcal{L}$ can be realized as a sublattice of $\{0,1\}|J(\mathcal{L})|+1$.

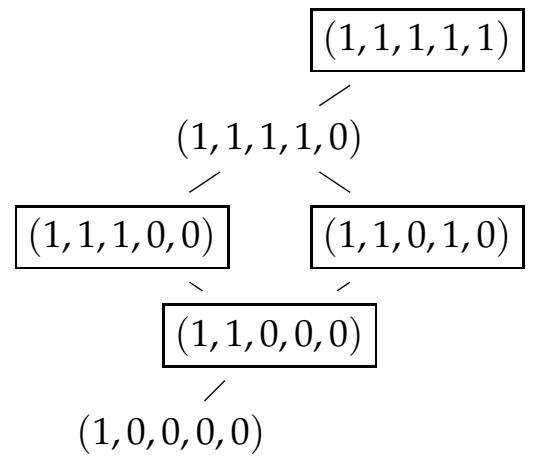

The lattice $\Pi_{2,4}$, with each element replaced by its image through $\pi$. 
3.5. Let $S$ be a commutative semigroup with identity. A 2-cocycle over $S$ is a function $\alpha: S \times S \longrightarrow \mathbb{R}^{\times}$such that $\alpha\left(s, s^{\prime}\right) \alpha\left(s+s^{\prime}, s^{\prime \prime}\right)=\alpha\left(s, s^{\prime}+s^{\prime \prime}\right) \alpha\left(s^{\prime}, s^{\prime \prime}\right)$ for all $s, s^{\prime}, s^{\prime \prime} \in S$. Given a 2-cocycle $\alpha$ over $S$, the $\alpha$-twisted semigroup algebra $\mathbb{k}^{\alpha}[S]$ is the associative $\mathbb{k}$ algebra whose underlying vector space has basis $\left\{X^{s} \mid s \in S\right\}$ and whose product over these generators is given by $X^{s} X^{t}=\alpha(s, t) X^{s+t}$. This is a noncommutative deformation of the classical semigroup algebra $\mathbb{k}[S]$. For more details see [RZ15, section 3]; in this reference the group is assumed to be cancellative, but this is not relevant for the definition of the twisted algebra.

Definition. Let $A$ be a connected $\mathbb{N}^{r}$-graded algebra. We say that $A$ is a quantum positive affine toric variety if there exist a positive affine semigroup $S$ and a 2-cocycle $\alpha$ over $S$ such that $A$ is isomorphic to the twisted semigroup algebra $\mathbb{k}^{\alpha}[S]$, and for each $S \in S \backslash\{0\}$ the element $X^{s}$ is homogeneous of nonzero degree with respect to the $\mathbb{N}^{r}$-grading of $\mathbb{k}^{\alpha}[S]$ induced by this isomorphism. In that case we refer to $S$ as the underlying semigroup of $A$.

Let $r \geq 1$ and let $\psi: S \longrightarrow \mathbb{N}^{r}$ be a monoid morphism such that $\psi^{-1}(0)=\{0\}$. The twisted semigroup algebra $\mathbb{k}^{\alpha}[S]$ can be endowed with a connected $\mathbb{N}^{r}$-grading setting $\operatorname{deg} X^{s}=\psi(s)$ for each $s \in S$. Conversely, any connected grading such that the elements of the form $X^{s}$ are homogeneous arises in this manner. In particular, if $A$ is a quantum positive toric variety with underlying semigroup $S$ then there is a corresponding monoid morphism $\psi: S \longrightarrow \mathbb{N}^{r}$, to which we will refer as the grading morphism.

Example. Let $q \in \mathbb{k}^{\times}$and set $\mathbf{q}=\left(\begin{array}{ccccc}1 & q & 1 & 1 & q \\ q^{-1} & 1 & q & q & q^{-2} \\ 1 & q^{-1} & 1 & 1 & q \\ 1 & q^{-1} & 1 & 1 & q \\ q^{-1} & q^{2} & q^{-1} & q^{-1} & 1\end{array}\right)$.

Let $\mathbb{k}_{\mathbf{q}}[X]$ be the twisted polynomial ring generated by variables $X_{1}, \ldots, X_{5}$ subject to the relations $X_{i} X_{j}=\mathbf{q}_{i, j} X_{j} X_{i}$. Given $t=(a, b, c, d, e) \in \mathbb{N}^{5}$ we set $X^{t}=X_{1}^{a} X_{2}^{b} X_{3}^{c} X_{4}^{d} X_{5}^{e}$. The formula $X^{t} X^{s}=\alpha(s, t) X^{t+s}$ induces a map $\alpha: \mathbb{N}^{5} \times \mathbb{N}^{5} \longrightarrow \mathbb{k}^{\times}$, and associativity of the product of $\mathbb{k}_{\mathbf{q}}[X]$ implies that $\alpha$ is a 2-cocycle. Recall from the examples in 3.2 and 3.4 that $S=\operatorname{str}\left(\Pi_{2,4}\right) \subset \mathbb{N}^{5}$ is an affine semigroup. The restriction of $\alpha$ to $S$ is also a 2-cocycle, and $\mathbb{k}^{\alpha}[S]$ is isomorphic to the subalgebra of $\mathbb{k}_{\mathbf{q}}[X]$ generated by the elements $X^{t}$ with $t$ running over the Hilbert basis of S. Setting

$$
\begin{array}{lll}
Y_{1}=X_{1} & Y_{2}=X_{1} X_{2} & Y_{3}=X_{1} X_{2} X_{3} \\
Y_{4}=X_{1} X_{2} X_{4} & Y_{5}=X_{1} X_{2} X_{3} X_{4} & Y_{6}=X_{1} X_{2} X_{3} X_{4} X_{5}
\end{array}
$$

it is routine to check that $Y_{j} Y_{i}=q^{-2} Y_{i} Y_{j}$ for $(i, j) \in\{(1,5),(2,4)\}$, that $Y_{2}$ and $Y_{3}$ commute, and that $Y_{j} Y_{i}=q^{-1} Y_{i} Y_{j}$ for all other possible pairs $i<j$. There is one more relation among these monomials, namely $Y_{2} Y_{3}=q^{-1} Y_{1} Y_{4}$. Thus $\mathbb{k}^{\alpha}[S]$ has as many generators as $S$, and can be presented by the commutation relations between them plus one extra relation arising from the fact that there is exactly one pair of incomparable elements in $\Pi_{2,4}$. We will see below in Proposition 3.14 that all quantum positive affine toric varieties and several related algebras have similar presentations. 
3.6. Quantum positive affine toric varieties were studied from the point of view of

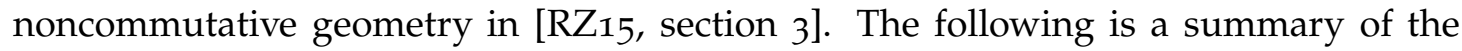
results proved there.

Proposition. Let $S$ be a positive affine semigroup and let $A$ be a positive quantum toric variety with underlying semigroup $S$. Then the following hold.

(a) $A$ is noetherian and integral.

(b) A has property $\chi$ and finite local dimension equal to the rank of $S$.

(c) Suppose $S$ is normal. Then $A$ is AS-Cohen-Macaulay and a maximal order in its division ring of fractions. Furthermore, $A$ is $A S$-Gorenstein if and only if there exists $s \in S$ such that relint $S=s+S$.

Proof. This is proved in [RZ15, section 3.2] in the case where the grading morphism $\psi$ is given by a full embedding of $S$ in $\mathbb{N}^{r}$ for some $r \geq 0$. The proposition follows from the fact that all the properties mentioned in it are independent of the grading, see paragraph 2.7

\section{Algebras with a quantum toric degeneration}

Classically, a toric degeneration of an algebraic variety $V$ is a flat deformation of $V$ into a toric variety $T$. Since varieties inherit many good properties from their flat deformations, and positive affine toric varieties are well studied, toric degeneration is a standard method to study algebraic varieties. With this in mind we introduce a noncommutative analogue of toric degeneration.

3.7 Definition. Let $A$ be a connected $\mathbb{N}^{r}$-graded algebra. We will say that $A$ has a quantum positive affine toric degeneration if it has a connected filtration such that its associated graded ring is a quantum positive affine toric variety. We refer to the underlying semigroup of this quantum positive affine toric variety as the semigroup associated to the degeneration.

For the sake of brevity we will write "quantum toric degeneration", omitting the adjectives "positive" and "affine". In view of Theorem 2.12 and Proposition 3.5, an algebra with a quantum toric degeneration is noetherian, integral, has property $\chi$ and finite local dimension. Furthermore, we can determine whether $A$ is AS-CohenMacaulay or AS-Gorenstein by studying the semigroup associated to the degeneration.

3.8. A standard technique for proving toric degeneration of a variety is to find the structure of a Hodge algebra in the coordinate ring of the variety. We now introduce a noncommutative notion, inspired in the definition of Hodge algebras and its descendants such as classical and quantum algebras with a straightening law, which will play a similar role for the rest of this article.

Definition. Let $A$ be a noetherian connected $\mathbb{N}^{r}$-graded algebra. Let $S$ be a positive affine semigroup and let $(\pi, P)$ be a presentation of $S$ as defined in 3.4 , with $P=\left\{\left(p_{i}, p_{i}^{\prime}\right) \mid 1 \leq i \leq\right.$ $m\}$. Let $\varphi: S \longrightarrow \mathbb{N}$ be a semigroup morphism such that $\varphi^{-1}(0)=\{0\}$, and set $\tilde{\varphi}=\varphi \circ \pi$.

We say that the algebra $A$ is of $(S, \varphi)$-type with respect to $(\pi, P)$ if the following hold. 
1. A is generated as algebra by a finite set of homogeneous elements $\left\{b_{1}, \ldots, b_{n}\right\}$ of the same cardinality as the Hilbert basis of $S$. We set $\mathbf{b}^{\xi}=\prod_{i=1}^{n} b_{i}^{\xi_{i}}$ for each $\xi \in \mathbb{N}^{n}$.

2. For each $1 \leq i<j \leq n$ and each $\xi \in \mathbb{N}^{n}$ such that $\tilde{\varphi}(\xi)<\varphi\left(s_{i}+s_{j}\right)$, there exist $c_{i, j} \in \mathbb{k}^{\times}$and $c_{\tilde{\xi}}^{i, j} \in \mathbb{k}$ such that

$$
b_{j} b_{i}=c_{i, j} b_{i} b_{j}+\sum_{\tilde{\varphi}(\xi)<\varphi\left(s_{i}+s_{j}\right)} c_{\tilde{\xi}}^{i, j} \mathbf{b}^{\xi} .
$$

3. For each $1 \leq i \leq m$ and each $\xi \in \mathbb{N}^{n}$ such that $\tilde{\varphi}(\xi)<\tilde{\varphi}\left(p_{i}\right)$ there exist $d_{i} \in \mathbb{k}^{\times}$and $d_{\xi}^{i} \in \mathbb{k}$ such that

$$
\mathbf{b}^{p_{i}^{\prime}}=d_{i} \mathbf{b}^{p_{i}}+\sum_{\tilde{\varphi}(\tilde{\xi})<\tilde{\varphi}\left(p_{i}\right)} d_{\tilde{\zeta}}^{i} \mathbf{b}^{\tilde{s}}
$$

We say that $A$ is of $(S, \varphi)$-type if there exists a presentation of $S$ such that $A$ is of $(S, \varphi)$-type with respect to it.

Remark. Since $\tilde{\varphi}\left(e_{i}\right)=\varphi\left(s_{i}\right)>0$ for all $i$, given $l \in \mathbb{N}$ there exist finitely many $\xi \in \mathbb{N}^{n}$ such that $\tilde{\varphi}(\xi) \leq l$ and so the sums on the right hand side of the formulas displayed in 2 and 3 are finite.

The reader familiar with Hodge algebras will notice that there is a condition missing in the definition, namely the existence of a set of linearly independent monomials on the generators. Thus the trivial algebra is of $(S, \varphi)$-type for any $S$ and $\varphi$. This omission will be revised in 3.10, where the existence of a linearly independent set will be shown to be equivalent to the existence of a quantum toric degeneration for the algebra.

Example. As stated above, this definition is inspired by that of quantum graded algebras with a straightening law (quantum graded ASL for short) as defined in [LR06, Definition 1.1.1]. A quantum graded ASL is not necessarily an algebra of $(S, \varphi)$-type, but the results from [RZ12, section 5] show that the quantized coordinate rings of grassmannians and their Richardson subvarieties have this structure. Our paradigmatic example is the quantum grassmannian.

Fix a field $\mathbb{k}$ and let $q \in \mathbb{k}^{\times}$. The quantum grassmannian $\mathcal{G}_{q}(2,4)$ is the algebra generated by elements $\left\{[i j],(i, j) \in \Pi_{2,4}\right\}$, subject to the commutation relations

$$
[34][12]=q^{-2}[12][34] \quad[24][13]=q^{-2}[13][24]+q^{-2}\left(q-q^{-1}\right)[12][34]
$$

while $Y X=q^{-1} X Y$ for any other pair $X<Y$ (here we identify the generating set with $\Pi_{2,4}$ in the obvious way). There is also a quantum Plücker relation, given by [14][23] = $q^{-1}[13][24]-q^{-2}[12][34]$. Notice that the set of generators can be identified with $\Pi_{2,4}$, and inherits the structure of a distributive lattice.

Setting wt as in [RZ12, Definition 4.3] we obtain an assignation
$[12] \mapsto 21$
$[13] \mapsto 12$
$[14] \mapsto 3$
$[23] \mapsto 11$
$[24] \mapsto 2$
$[34] \mapsto 1$ 
which extends to a semigroup morphism $\varphi: \operatorname{str}\left(\Pi_{2,4}\right) \longrightarrow \mathbb{N}$. It is now a matter of routine computations to check that $\mathcal{G}_{q}(2,4)$ is of $\left(\operatorname{str}\left(\Pi_{2,4}\right), \varphi\right)$-type.

3.9. If $A$ is an algebra of $(S, \varphi)$-type we write $F_{l} A=\left\langle\mathbf{b}^{\xi} \mid \tilde{\varphi}(\xi) \leq l\right\rangle$ for each $l \in \mathbb{N}$. The fact that $\tilde{\varphi}\left(e_{i}\right)>0$ for all $i$ implies that this is a finite dimensional vector space, and that $F_{0} A=\mathbb{k}$. The following lemma shows that $\mathcal{F}=\left\{F_{l} A\right\}_{l \geq 0}$ is a connected filtration on $A$.

Lemma. Let $S$ be a positive affine semigroup. Let $A$ be a noetherian connected $\mathbb{N}^{r}$-graded algebra, and assume that it is of $(S, \varphi)$-type with respect to a presentation $(\pi, P)$. Then the following hold.

1. Given $\xi, v \in \mathbb{N}^{n}$ there exists $c_{\xi, v} \in \mathbb{k}^{\times}$such that $\mathbf{b}^{\tilde{b}} \mathbf{b}^{v} \equiv c_{\xi, v} \mathbf{b}^{\tilde{\xi}+v} \bmod F_{\tilde{\varphi}(\xi+v)-1} A$. In particular $\mathcal{F}$ is a filtration on $A$.

2. Given $(\xi, v) \in L(\pi)$ there exists $d_{\xi, v} \in \mathbb{k}^{\times}$such that $\mathbf{b}^{\xi} \equiv d_{\xi, v} \mathbf{b}^{v} \bmod F_{\tilde{\varphi}(\xi)-1} A$.

Proof. To prove item 1 we proceed by induction on $\tilde{\varphi}(\xi+v)$, with the 0 -th step being obvious since $F_{0} A=\mathbb{k}$ is a subalgebra of $A$. Suppose that the result holds for all $l<\tilde{\varphi}(\xi+v)$ and let $i$ be the least integer such that $\xi_{i} \neq 0$, so $\mathbf{b}^{\tau} \mathbf{b}^{v}=b_{i}\left(\mathbf{b}^{\tilde{\xi}-e_{i}} \mathbf{b}^{v}\right)$. Using the inductive hypothesis we obtain

$$
\begin{aligned}
b_{i}\left(\mathbf{b}^{\tilde{\xi}-e_{i}} \mathbf{b}^{v}\right) & =b_{i}\left(c_{\xi-e_{i}, \nu} \mathbf{b}^{\xi-e_{i}+v}+\sum_{\tilde{\varphi}(\rho)<\tilde{\varphi}\left(\xi-e_{i}+v\right)} c_{\rho} \mathbf{b}^{\rho}\right) \\
& =c_{\xi}-e_{i}, \nu b_{i} \mathbf{b}^{\xi-e_{i}+v}+\sum_{\tilde{\varphi}(\rho)<\tilde{\varphi}\left(\xi-e_{i}+v\right)} c_{\rho} b_{i} \mathbf{b}^{\rho}
\end{aligned}
$$

where the $c_{\rho} \in \mathbb{k}$ and $c_{\xi-e_{i}, v} \in \mathbb{k}^{\times}$. The inductive hypothesis also implies each product $b_{i} \mathbf{b}^{\rho}$ lies in $F_{\tilde{\varphi}(\xi+v)-1} A$, so

$$
b_{i} \mathbf{b}^{\tilde{\xi}-e_{i}} \mathbf{b}^{v} \equiv c_{\tilde{\xi}-e_{i}, \nu} b_{i} \mathbf{b}^{\tilde{\xi}-e_{i}+v} \bmod F_{\tilde{\varphi}(\xi+v)-1} A .
$$

Now let $j$ be the least integer such that $v_{j} \neq 0$. If $i \leq j$ then $b_{i} \mathbf{b}^{\tilde{\xi}-e_{i}+v}=\mathbf{b}^{\tilde{\xi}+v}$ and we are finished; otherwise, using item 2 of Definition 3.8 and a similar argument as before, we obtain

$$
b_{i} \mathbf{b}^{\xi-e_{i}+v}=\left(b_{i} b_{j}\right)\left(\mathbf{b}^{\xi-e_{i}+v-e_{j}}\right) \equiv c_{j, i}\left(b_{j} b_{i}\right)\left(\mathbf{b}^{\tilde{\xi}-e_{i}+v-e_{j}}\right) \quad \bmod F_{\tilde{\varphi}(\xi+v)-1} A
$$

The same reasoning applied to the product $b_{j}\left(b_{i} \mathbf{b}^{\xi-e_{i}+v-e_{j}}\right)$ shows that

$$
b_{j}\left(b_{i} \mathbf{b}^{\xi-e_{i}+v-e_{j}}\right) \equiv c_{e_{i}, \xi-e_{i}+v-e_{j}} b_{j}\left(\mathbf{b}^{\tilde{s}+v-e_{j}}\right) \quad \bmod F_{\tilde{\varphi}(\xi+v)-1} A .
$$

Since $j<i$, the definition of $j$ implies $b_{j}\left(\mathbf{b}^{\xi+v-e_{j}}\right)=\mathbf{b}^{\xi+v}$, so the proof of item $\square$ is complete.

We now prove item 2 , Set

$$
T=\left\{(\xi, v) \in L(\pi) \mid \mathbf{b}^{\xi} \equiv c \mathbf{b}^{v} \quad \bmod F_{\tilde{\varphi}(\xi)-1} \text { for some } c \in \mathbb{k}^{\times}\right\} .
$$


We will show that $L(\pi) \subset T$, which clearly implies the desired result. By definition $T$ is an equivalence relation, and item 1 implies it is a congruence on $\mathbb{N}^{n}$. By item 3 of Definition 3.8, every pair $\left(p_{i}, p_{i}^{\prime}\right) \in P$ lies in $T$. Since $L(\pi)$ is the smallest congruence containing $P$, we deduce that $L(\pi) \subset T$.

3.10. Let $S$ be a positive affine semigroup and let $(\pi, P)$ be a presentation of $S$. A section of $\pi$ is a function $\tau: S \longrightarrow \mathbb{N}^{n}$ such that $\pi \circ \tau=\operatorname{Id}_{S}$, that is $s=\sum_{i} \tau(s)_{i} s_{i}$ for every $s \in S$. If $A$ is an algebra of $(S, \varphi)$-type then Lemma 3.9 implies that for any section $\tau$ of $\pi$ the set $\left\{\mathbf{b}^{\tau(s)} \mid s \in S\right\}$ spans $A$. We now show that an algebra has a quantum toric degeneration with associated semigroup $S$ if and only if it is of $(S, \varphi)$ type for an adequate morphism $\varphi$ and the spanning set determined by any section is linearly independent.

Proposition. Let $S$ be a positive affine semigroup, and let $A$ be a noetherian connected $\mathbb{N}^{r}$ graded algebra. The following statements are equivalent.

1. The algebra A has a quantum toric degeneration with associated semigroup $S$.

2. For every presentation $(\pi, P)$ of $S$ there exists a semigroup morphism $\varphi: S \longrightarrow \mathbb{N}$ such that $A$ is of $(S, \varphi)$-type with respect to $(\pi, P)$, and for every section $\tau: S \longrightarrow \mathbb{N}^{n}$ of $\pi$ the set $\left\{\mathbf{b}^{\tau(s)} \mid s \in S\right\}$ is linearly independent.

3. There exist a presentation $(\pi, P)$, a semigroup morphism $\varphi: S \longrightarrow \mathbb{N}$ and a section $\tau: S \longrightarrow \mathbb{N}^{n}$ of $\pi$ such that $A$ is of $(S, \varphi)$-type with respect to $(\pi, P)$ and the set $\left\{\mathbf{b}^{\tau(s)} \mid s \in S\right\}$ is linearly independent.

Proof. We first show that 1 implies 2. By hypohtesis there exists a filtration by graded subspaces $\mathcal{F}=\left\{F_{l} A\right\}_{l \geq 0}$ such that $\operatorname{gr}_{\mathcal{F}} A \cong \mathbb{k}^{\alpha}[S]$ as $\mathbb{N}^{r+1}$-graded algebras for some 2-cocycle $\alpha: S \times S \longrightarrow \mathbb{k}^{\times}$, with the grading on the twisted semigroup algebra given by a semigroup morphism $\psi: S \longrightarrow \mathbb{N}^{r+1}$ such that $\psi^{-1}(0)=\{0\}$. We identify $\operatorname{gr}_{\mathcal{F}} A$ with $\mathbb{k}^{\alpha}[S]$ through this isomorphism to simplify notation.

Fix a presentation $(\pi, P)$ of $S$. For each $1 \leq i \leq n$ we choose homogeneous elements $b_{i} \in A$ such that $\mathrm{gr} b_{i}=X^{s_{i}}$. By definition of the product of an associated graded ring, for each $\xi \in \mathbb{N}^{n}$ the element $\prod_{i=1}^{n}\left(\operatorname{gr} b_{i}\right)^{\xi_{i}}$ equals either $\operatorname{gr}\left(\prod_{i=1}^{n} b_{i}^{\xi_{i}}\right)$ or zero. Since $\mathbb{K}^{\alpha}[S]$ is an integral ring the last possibility cannot occur, so $\operatorname{gr}\left(\mathbf{b}^{\tilde{\xi}}\right)$ equals a nonzero multiple of $X^{\pi(\tilde{\zeta})}$. Thus if $\tau: S \longrightarrow \mathbb{N}^{n}$ is a section of $\pi$ then for each $s \in S$ there exists a nonzero constant $c_{S}$ such that $\operatorname{gr} b^{\tau(s)} \equiv c_{S} X^{s}$, and so the set $\left\{\operatorname{gr} \mathbf{b}^{\tau(s)} \mid s \in S\right\}$ is a basis of $\mathbb{k}^{\alpha}[S]$, which implies that $\left\{\mathbf{b}^{\tau(s)} \mid s \in S\right\}$ is a basis of $A$. This also proves that $A$ satisfies item 1 of Definition 3.8 .

Let $\varphi: S \longrightarrow \mathbb{N}$ be the additive map given by $s \mapsto \psi(s)_{r+1}$; equivalently $\varphi(s)$ is the minimal $l$ such that $\mathbf{b}^{\xi} \in F_{l} A$ for all $\xi \in \pi^{-1}(s)$. In particular $\varphi\left(s_{i}\right)>0$ since $F_{0} A=\mathbb{k}$. Also $F_{l} A=\left\langle\mathbf{b}^{\xi} \mid \tilde{\varphi}(\xi) \leq l\right\rangle$, and since for each $\xi \in \mathbb{N}^{n}$ there exists a nonzero constant $c_{\xi}$ such that $\operatorname{gr} b^{\tau} \equiv c_{\xi} \operatorname{gr} b^{\tau(\pi(\xi))}$ we actually have $F_{l} A=\left\langle\mathbf{b}^{\tau(s)} \mid \varphi(s) \leq l\right\rangle$. Finally, for 
each $1 \leq i, j \leq n$ and each $1 \leq k \leq m$ there exist $c_{i, j}, d_{k} \in \mathbb{k}^{\times}$such that

$$
\begin{aligned}
\operatorname{gr}\left(b_{j} b_{i}\right) & =c_{i, j} \operatorname{gr}\left(b_{i} b_{j}\right), \\
\operatorname{gr} \mathbf{b}^{p_{k}^{\prime}} & =d_{k} \operatorname{gr} \mathbf{b}^{p_{k}}
\end{aligned}
$$

hold in $\operatorname{gr}_{\mathcal{F}} A$, which implies that items 2 and 3 of Definition 3.8 hold in $A$ for the morphism $\varphi$ we have just defined. Thus $A$ is of $(S, \varphi)$-type, and we have proved 1 implies 2 ,

We said in 3.4 that every positive affine semigroup has a presentation so clearly 2 implies 3. Let us see that 3 implies 1, Define the filtration $\mathcal{F}=\left\{F_{l} A\right\}_{l \geq 0}$ as in 3.9. By item 2 of Lemma 3.9, the set $\left\{\mathbf{b}^{\tau(s)} \mid \varphi(s) \leq l\right\}$ generates $F_{l} A$ for each $l \in \mathbb{N}$, and since by hypothesis it is linearly independent, it is a basis of $F_{l} A$. Hence $\operatorname{gr}_{\mathcal{F}} A$ is generated by $\left\{\operatorname{grb}^{\tau(s)} \mid s \in S\right\}$. Once again by Lemma 3.9 for each $s, s^{\prime} \in S$ there exist $\beta\left(s, s^{\prime}\right), \alpha\left(s, s^{\prime}\right) \in \mathbb{k}^{\times}$such that

$$
\left(\operatorname{gr} \mathbf{b}^{\tau(s)}\right)\left(\operatorname{gr} \mathbf{b}^{\tau\left(s^{\prime}\right)}\right)=\beta\left(s, s^{\prime}\right) \operatorname{gr} \mathbf{b}^{\tau(s)+\tau\left(s^{\prime}\right)}=\alpha\left(s, s^{\prime}\right) \operatorname{gr} \mathbf{b}^{\tau\left(s+s^{\prime}\right)} .
$$

Associativity of the product of $\operatorname{gr}_{\mathcal{F}} A$ implies that $\alpha: S \times S \longrightarrow \mathbb{k}^{\times}$is a 2-coycle, so we may consider the $\mathbb{k}$-linear map $\mathbb{k}^{\alpha}[S] \longrightarrow \operatorname{gr}_{\mathcal{F}} A$ induced by the assignation $X^{s} \mapsto \operatorname{gr} \mathbf{b}^{\tau(s)}$, which is a multiplicative map. Since $S$ is positive we must have $\tau(0)=0$, and hence $\alpha(s, 0)=\alpha(0, s)=1$ for all $s \in S$ which implies that our multiplicative map is unitaty and hence an isomorphism of $\mathbb{k}$-algebras. Furthermore, the elements $\operatorname{gr} \mathbf{b}^{\tau(s)}$ are homogeneous, so this algebra is indeed a quantum positive affine toric variety.

Example. We return one last time to the example of the quantum grassmannian $\mathcal{G}_{q}(2,4)$ over an arbitrary field discussed in 3.8 . We extend the order of $\Pi_{2,4}$ to a total order so we can identify the free abelian semigroup over $\Pi_{2,4}$ with $\mathbb{N}^{6}$, and let $\pi: \mathbb{N}^{6} \longrightarrow \operatorname{str}\left(\Pi_{2,4}\right)$ be the presentation morphism described in 3.4. For each $s \in \operatorname{str}\left(\Pi_{2,4}\right)$ the fiber $\pi^{-1}(s)$ is finite and has a unique maximal element with respect to the total lexicographic order of $\mathbb{N}^{6}$, which we denote by $\tau(s)$. The monomials corresponding to this section are precisely the standard monomials introduced in [LRo6, Definition 3.2.1]. Using the map $\varphi$ from 3.8 the quantum toric variety obtained by degeneration is the twisted semigroup algebra presented in 3.5 (this explains the rather odd choice of commutation coefficients in that example).

A similar argument holds not just for quantum grassmannians, but for the large class of quantum graded ASL satisfying condition (C) introduced in [RZ12, Definition 4.1]. This includes all quantum grassmannians in type A, along with their Schubert and Richardson subvariaties. In the following section we will show that quantized coordinate rings of Schubert subvarieties of arbitrary flag varieties also have a quantum toric degeneration.

3.11. We now introduce a second notion related to quantum toric degenerations. Recall that a commutative semigroup $S$ is said to be well-ordered if there exists a wellorder $<$ on $S$ compatible with the additive structure, i.e. such that for all $s, s^{\prime}, s^{\prime \prime} \in S$ the inequality $s<s^{\prime}$ implies $s+s^{\prime \prime}<s^{\prime}+s^{\prime \prime}$.

Definition. Let $S$ be a commutative semigroup, and let $<$ be a well-order on $S$ compatible with the semigroup structure. Let $A$ be a connected $\mathbb{N}^{l}$-graded algebra for some $l \in \mathbb{N}$. An 
$(S,<)$-basis for $A$ is an ordered basis $\left\{b_{s} \mid s \in S\right\}$ consisting of homogeneous elements, such that for all $s, s^{\prime}, s^{\prime \prime} \in S$ with $s^{\prime \prime}<s+s^{\prime}$ there exist $c_{s, s^{\prime}} \in \mathbb{k}^{\times}$and $c_{s, s^{\prime}}^{s^{\prime \prime}} \in \mathbb{R}$ such that

$$
b_{s} b_{s^{\prime}}=c_{s, s^{\prime}} b_{s+s^{\prime}}+\sum_{s^{\prime \prime}<s+s^{\prime}} c_{s, s^{\prime}}^{s^{\prime \prime}} b_{s^{\prime \prime}}
$$

3.12. Let $S$ be a commutative semigroup with neural element 0 . Assume $<$ is a wellorder on $S$ compatible with its additive structure, and let $A$ be an algebra. An $(S,<)$ filtration on $A$ is a collection of vector spaces $\mathcal{F}=\left\{F_{s} A \mid s \in S\right\}$, such that $F_{s} A \cdot F_{s^{\prime}} A \subset$ $F_{s+s^{\prime}} A$ for all $s, s^{\prime} \in S$, and such that $F_{s} A \subseteq F_{s^{\prime}} A$ whenever $s \leq s^{\prime}$. The standard notions related to $\mathbb{N}$-filtrations translate easily to the context of $(S,<)$-filtrations. We will assume that our filtrations are always exhaustive, so $A=\bigcup_{s \in S} F_{S} A$, and discrete, so $F_{s} A=0$ for all $s<0$.

Let $A$ be an $S$-filtered algebra. The associated graded algebra $\operatorname{gr}_{\mathcal{F}} A$ is defined setting $F_{<s} A=\sum_{t<s} F_{t} A$ and taking

$$
\operatorname{gr}_{\mathcal{F}} A=\bigoplus_{s \in S} \frac{F_{S} A}{F_{<s} A}
$$

As usual, for each element $a \in A$ we may define gr $a$ as the image of $a$ in the quotient $F_{S} A / F_{<s} A$ where $s$ is the first element of $S$ such that $a \in F_{S} A$; notice that this element exists because $<$ is a well-order. The product can then be defined as in the $\mathbb{N}$-filtered case, namely if $a, b \in A$ and $s, t$ are minimal elements such that $a \in F_{s} A$ and $b \in F_{t} A$, then $(\operatorname{gr} a)(\operatorname{gr} b)$ equals the image of $a b$ in $F_{s+t} A / F_{<s+t} A$, which equals $\operatorname{gr}(a b)$ if $t+s$ is minimal with respect to the property that $a b \in F_{t+s} A$ and zero otherwise.

Lemma. Let $S$ be a positive affine semigroup and let $<$ be a well-order of $S$ compatible with the semigroup structure. Let $A$ be a noetherian $\mathbb{N}^{l}$-graded connected algebra for some $l \in \mathbb{N}$, and assume $A$ has an $(S,<)$-basis $\mathcal{B}=\left\{b_{s} \mid s \in S\right\}$. Set $F_{s} A=\left\langle b_{t} \mid t \leq s \in S\right\rangle$ and $\mathcal{F}=\left\{F_{S} A \mid s \in S\right\}$. Also let $\left\{s_{1}, \ldots, s_{n}\right\}$ be the Hilbert basis of $S$ and set $b_{i}=b_{s_{i}}$ for all $1 \leq i \leq n$. The following hold.

1. The family $\mathcal{F}$ is an $(S,<)$-filtration. Furthermore, each quotient $F_{S} A / F_{<s} A$ is of dimension 1 and $F_{0} A=\langle 1\rangle$.

2. There exists a 2-cocycle $\alpha$ over $S$ such that $\operatorname{gr}_{\mathcal{F}} A$ is isomorphic as S-graded algebra to $\mathbb{K}^{\alpha}[S]$ for the obvious S-grading on this.

3. The algebra $A$ is generated by the set $\left\{b_{i} \mid 1 \leq i \leq n\right\}$.

Proof. The fact that $\mathcal{F}$ is an exhaustive and discrete $(S,<)$-filtration is an immediate consequence of the definition of an $(S,<)$-basis. Also $F_{<s} A=\left\langle b_{t} \mid t<s\right\rangle$, so $F_{s} A / F_{<s} A$ is generated by the image of $b_{s}$ in the quotient, which is nonzero. Finally, writing 1 as a linear combination of the $b_{s}$ and using a leading term argument, it is easy to see that $1 \in F_{0} A$ and hence it must generate it. Notice that this implies that $b_{0}$ is a scalar so without loss of generality we may assume that $b_{0}=1$. This proves item 1 . 
Set $\alpha\left(s, s^{\prime}\right)=c_{s, s^{\prime}}$ for each $s, s^{\prime} \in S$. By the first item the set $\left\{\operatorname{gr} b_{s} \mid s \in S\right\}$ is a basis of $\operatorname{gr}_{\mathcal{F}} A$, and $\left(\operatorname{gr} b_{s}\right)\left(\operatorname{gr} b_{s^{\prime}}\right)=c_{s, s^{\prime}}$ gr $b_{s+s^{\prime}}$ by definition of the product on the associated graded ring. Associativity of the product in $\operatorname{gr}_{\mathcal{F}} A$ implies then that $\alpha$ is a 2-cocycle over $S$, and furthermore the map $\operatorname{gr}_{\mathcal{F}} A \longrightarrow \mathbb{k}^{\alpha}[S]$ sending $\operatorname{gr} b_{S}$ to $X^{s}$ is a multiplicative $S$-graded vector-space isomorphism. Since we are assuming that $b_{0}=1$, it follows that $\alpha(1, s)=\alpha(s, 1)=1$ for all $s \in S$ and hence our isomorphism preserves the unit, and is thus a ring isomorphism. This proves item 2.

Finally, in order to prove that the $b_{i}$ 's generate $A$ it is enough to show that each $b_{s}$ is in the algebra generated by these elements. Suppose this is not the case. Then, since $S$ is well-ordered by $<$, there exists a minimal $s$ such that $b_{s}$ is not in the algebra generated by the $b_{i}$ 's. Take $\xi=\left(\xi_{1}, \ldots, \xi_{n}\right) \in \mathbb{N}^{n}$ such that $\sum_{i} \xi_{i} s_{i}=s$. By the definition of the product in the associated graded ring, $\prod_{i}\left(\operatorname{gr} b_{i}\right)^{\xi_{i}}$ equals either $\operatorname{gr}\left(\mathbf{b}^{\tau}\right)$ or zero, and since $\operatorname{gr}_{\mathcal{F}} A \cong \mathbb{k}^{\alpha}[S]$ is integral (see [RZ15, Lemma 3.2.3]) the second possibility can not occur. Thus $\operatorname{gr}\left(\mathbf{b}^{\xi}\right)$ is a nonzero element $\operatorname{of} \operatorname{gr}_{\mathcal{F}} A$ of degree $\pi(\xi)$, so item 1 of this lemma implies that $\operatorname{gr} b_{s}=c \operatorname{gr}\left(\mathbf{b}^{\xi}\right)$ for some $c \in \mathbb{k}^{\times}$, and hence $b_{s}=c \mathbf{b}^{\tau}+$ $\sum_{t<s} c_{t} b_{t}$. By the minimality of $s$ all the $b_{t}$ 's appearing in the sum on the right hand side of the equation lie in the algebra generated by the $b_{i}{ }^{\prime} \mathrm{s}$, and clearly so does $\mathbf{b}^{\tau}$, a contradiction.

3.13. There is an obvious way to obtain well-orderings on positive affine semigroups. Since $S$ is a positive affine semigroup it can be embedded in $\mathbb{N}^{r}$ for some $r \geq 0$ through a monoid morphism $\iota: S \longrightarrow \mathbb{N}^{r}$. Now $\mathbb{N}^{r}$ is a well-ordered semigroup with the lexicographic order, which is compatible with its additive structure, so we may pullback the lexicographic order through $\iota$ and thus obtain a well-order $<^{l}$ over $S$, which is also compatible with its additive structure. Notice that in this case 0 is always the minimal element of $S$.

Proposition. Let $S$ be a positive affine semigroup, and let $A$ be a noetherian connected $\mathbb{N}^{l}$ graded algebra for some $l \in \mathbb{N}$. The algebra $A$ has a quantum affine toric degeneration with underlying semigroup $S$ if and only if there exists an embedding $\iota: S \longrightarrow \mathbb{N}^{t}$ such that $A$ has an ordered $\left(S,<^{l}\right)$-basis.

Proof. Suppose $A$ has a quantum affine toric degeneration with underlying semigroup $S$. Then by Proposition 3.10 there exists a semigroup morphism $\varphi: S \longrightarrow \mathbb{N}$ such that $A$ is of $(S, \varphi)$-type, and we may choose any section $\tau: S \longrightarrow \mathbb{N}^{n}$ to obtain a basis $\mathcal{B}=\left\{\mathbf{b}^{\tau(s)} \mid s \in S\right\}$. Let $\rho: S \longrightarrow \mathbb{N}^{t-1}$ be an embedding $(t>1)$ and let $\iota: S \longrightarrow \mathbb{N}^{t}$ be defined as $\iota(s)=(\varphi(s), \rho(s))$, which is an embedding of $S$ since $\rho$ is an embedding. Write $<$ for $<^{\prime}$, and notice that $\varphi(s)<\varphi\left(s^{\prime}\right)$ implies $s<s^{\prime}$. By Lemma 3.9. for all $s, s^{\prime} \in S$ and all $s^{\prime \prime}$ such that $\varphi\left(s^{\prime \prime}\right)<\varphi\left(s+s^{\prime}\right)$ there exist $c_{s, s^{\prime}} \in \mathbb{k}^{\times}$and $c_{s, s^{\prime}}^{s^{\prime \prime}} \in \mathbb{k}$ such that

$$
\mathbf{b}^{\tau(s)} \mathbf{b}^{\tau\left(s^{\prime}\right)}=c_{s, s^{\prime}} \mathbf{b}^{\tau\left(s+s^{\prime}\right)}+\sum_{\varphi\left(s^{\prime \prime}\right)<\varphi\left(s+s^{\prime}\right)} c_{s, s^{\prime}}^{s^{\prime \prime}} \mathbf{b}^{\tau\left(s^{\prime \prime}\right)},
$$

which implies $\mathcal{B}$ is an ordered $S$-basis with respect to $<$.

Now assume $A$ has an ordered $S$-basis with respect to some total order $<$ induced by an embedding $\iota: S \longrightarrow \mathbb{N}^{t}$. Since $<$ is the pull-back of the lexicographic order 
through an embedding, we might as well assume $S \subset \mathbb{N}^{t}$ and that $<$ is the lexicographic order. By the previous lemma, we already know that the $b_{i}{ }^{\prime}$ s generate $A$, so all that is left to do is to prove the existence of an additive map $\varphi: S \longrightarrow \mathbb{N}$ and that the desired relations hold.

As before, we denote by $\pi: \mathbb{N}^{n} \longrightarrow S$ the map $\left(\xi_{1}, \ldots, \xi_{n}\right) \mapsto \sum_{i} \xi_{i} s_{i}$. Recall that using the $(S,<)$-filtration $\mathcal{F}$ defined in the previous lemma, we proved that $\operatorname{gr}_{\mathcal{F}} A \cong$ $\mathbb{k}^{\alpha}[S]$ for some 2 -cocycle $S$. This implies that for all $1 \leq i<j \leq n$ and all $\xi \in \mathbb{N}^{n}$ such that $\pi(\xi)<s_{i}+s_{j}$ there exist $c_{i, j} \in \mathbb{k}^{\times}$and $c_{\tilde{\xi}}^{i, j} \in \mathbb{k}$ such that

$$
b_{j} b_{i}=c_{i, j} b_{i} b_{j}+\sum_{\pi(\xi)<\pi\left(s_{i}+s_{j}\right)} c_{\xi}^{i, j} \mathbf{b}^{\xi},
$$

and for each $1 \leq i \leq m$ and each $\xi \in \mathbb{N}^{n}$ such that $\pi(\xi)<p_{i}$ there exist $d_{i} \in \mathbb{k}^{\times}$and $d_{\xi}^{i} \in \mathbb{k}$ such that

$$
\mathbf{b}^{p_{i}^{\prime}}=d_{i} \mathbf{b}^{p_{i}}+\sum_{\pi(\xi)<p_{i}} d_{\zeta}^{i} \mathbf{b}^{\xi}
$$

Let $C \subset S$ be the set consisting of the following elements:

- all $s_{i}+s_{j}$ with $1 \leq i<j \leq n$;

- all $\pi\left(p_{i}\right)$ with $1 \leq i \leq m$;

- all $\pi(\xi)$ such that $c_{\tilde{\xi}}^{i, j} \neq 0$ for some $1 \leq i<j \leq n$;

- and all $\pi(\xi)$ such that $d_{\tilde{\zeta}}^{i} \neq 0$ for some $1 \leq i \leq m$.

The set $C$ is finite and hence is contained in a cube $[0, N]^{t}$ for $N$ large enough. Set $\varphi$ : $\mathbb{N}^{t} \longrightarrow \mathbb{N}$ to be the semigroup morphism defined by $\left(c_{1}, \ldots, c_{t}\right) \mapsto \sum_{i=1}^{t} c_{i}(N+1)^{t-i}$. If $c \in C$ then $\varphi(c)$ is the unique natural number such that its $N+1$-adic expansion has $c_{i}$ as its $i$-th digit. This implies that $\varphi$ respects the restriction of the lexicographic order to $C$, and thus $A$ is of $(S, \varphi)$-type.

Let $\tau: S \longrightarrow \mathbb{N}^{n}$ be any section of $\pi$. The algebra $\operatorname{gr}_{\mathcal{F}} A$ has a natural $S$-grading, and for each $s \in S$ the element $\operatorname{gr} \mathbf{b}^{\tau(s)}$ is of degree $s$. As we have already observed, this is a non-zero element so the set $\left\{\operatorname{gr}^{\tau(s)} \mid s \in S\right\}$ is a basis of $\operatorname{gr}_{\mathcal{F}} A$, which implies that $\left\{\mathbf{b}^{\tau(s)} \mid s \in S\right\}$ is a basis of $A$. Thus by Proposition $3.10 A$ has a quantum affine toric degeneration with underlying semigroup $S$.

Remark. The trick of turning the S-filtration into an $\mathbb{N}$-filtration using $N+1$-adic expansions is due to Caldero [Calo2, Lemma 3.2]. A similar though less general version of this idea appears in [GL96] and [RZ12].

3.14. We finish this section with an easy consequence of Lemma 3.9. It will not be used in the sequel, but we include it for completeness. 
Proposition. Let $S$ be a positive affine semigroup and $A$ a noetherian connected $\mathbb{N}^{r}$ - graded algebra. If $A$ is of $(S, \varphi)$-type for some monoid morphism $\varphi: S \longrightarrow \mathbb{N}$, and there exists some section $\tau$ of $\pi$ such that the set $\left\{\mathbf{b}^{\tau(s)} \mid s \in S\right\}$ is linearly independent, then the relations given in items 2 and 3 of Definition 3.8 give a presentation of $A$.

Proof. Since $A$ is of $(S, \varphi)$-type, it is generated as algebra by homogeneous elements $b_{1}, \ldots, b_{n}$, and there exist constants $c_{i, j}, c_{\tilde{\xi}}^{i, j}, d_{i}, d_{\tilde{\xi}}^{i}$ such that $A$ complies with Definition 3.8 Furthermore, the relations described in items 2 and 3 of this definition are homogeneous.

Let $B$ be the free algebra generated by $X_{1}, \ldots, X_{n}$ and let $I$ be the ideal of $B$ generated by the elements

$$
\begin{array}{lr}
X_{j} X_{i}-c_{i, j} X_{i} X_{j}-\sum_{\tilde{\varphi}(\xi)<\varphi\left(s_{i}+s_{j}\right)} c_{\tilde{\xi}}^{i, j} X^{\xi}, & \text { for } 1 \leq i<j \leq n ; \\
X^{p_{i}^{\prime}}-d_{i} X^{p_{i}}-\sum_{\tilde{\varphi}(\xi)<\tilde{\varphi}\left(p_{i}\right)} d_{\tilde{\xi}}^{i} X^{\xi}, & \text { for } 1 \leq i \leq m ;
\end{array}
$$

where $X^{\xi}=X_{1}^{\xi_{1}} X_{2}^{\xi_{2}} \cdots X_{n}^{\zeta_{n}}$ for each $\xi \in \mathbb{N}^{n}$. We put an $\mathbb{N}^{r}$ grading on $B$ by setting $\operatorname{deg} X_{i}=\operatorname{deg} b_{i}$, and this induces an $\mathbb{N}^{r}$ grading on $B / I$. Since $B$ is a free algebra the assignation $X_{i} \mapsto b_{i}$ induces a morphism of graded algebras $B \longrightarrow A$, which factors through $B / I$. We thus obtain a morphism of $\mathbb{N}^{r}$-graded algebras $f: B / I \longrightarrow A$.

We denote by $Y_{i}$ the image of $X_{i}$ in $B / I$. Clearly $B / I$ is an $(S, \varphi)$ - algebra, and the algebra map $f: B / I \longrightarrow A$ sends $Y_{i}$ to $b_{i}$ for all $i$. Since $f\left(Y^{\tau(s)}\right)=\mathbf{b}^{\tau(s)}$, the set $\left\{Y^{\tau(s)} \mid s \in S\right\}$ is linearly independent and hence a basis of $B / I$. Thus $f$ maps a basis onto a basis, so it is an isomorphism.

\section{Quantum affine toric degeneration of quantum Schubert va- rieties}

We apply the results in the previous section to study Schubert varieties of quantum flag varieties. We recall the definitions of quantum flag and Schubert varieties with some detail in order to establish notation. We then adapt an argument due to P. Caldero to show that these algebras have $(S,<)$-bases for adequate semigroups $S$. The main ingredient in the construction of these bases is the canonical or global basis of $U_{q}^{-}(\mathfrak{g})$ discovered independently by Lusztig and Kashiwara. The semigroup arises out of the string parametrization of this basis.

\section{Quantum flag and Schubert varieties}

4.1. Let $\mathfrak{g}$ be a complex semisimple Lie algebra. We denote by $\Phi$ the root system of $\mathfrak{g}$ with respect to a fixed Cartan subalgebra, and by $\mathbb{Z} \Phi$ its root lattice. We also fix a basis $\Pi \subset \Phi$ of positive roots, and write $P=\left\{\omega_{\alpha} \mid \alpha \in \Pi\right\}$ for the set of corresponding fundamental weights. We denote by $\Lambda$ the weight lattice $\sum_{\alpha \in \Pi} \mathbb{Z} \omega_{\alpha}$ and by $\Lambda^{+}$the set of dominant integral weights $\sum_{\alpha \in \Pi} \mathbb{N} \omega_{\alpha}$. 
Let $W$ be the Weyl group of $\mathfrak{g}$, and $s_{\alpha} \in W$ the reflection corresponding to $\alpha \in \Pi$. Given an element $w \in W$ we denote its length by $\ell(w)$, and set $N$ to be the length of $w_{0}$, the longest element of $W$. A decomposition of $w \in W$ is a word on the generators $s_{\alpha}$ that equals $w$ in $W$. The decomposition is reduced if it is of minimal length, i.e. its length equals $\ell(w)$. We denote by $(-,-)$ the standard $W$-invariant pairing between $\mathbb{Z} \Phi$ and $\Lambda$, and write $\langle\lambda, \alpha\rangle=\frac{2(\lambda, \alpha)}{(\alpha, \alpha)}$ for all $\lambda \in \Lambda$ and $\alpha \in \Phi$, so if $\lambda=\sum_{\alpha \in \Pi} r_{\alpha} \omega_{\alpha}$ then $\langle\lambda, \alpha\rangle=r_{\alpha}$.

4.2. Fix $q \in \mathbb{k}^{\times}$. Let $U_{q}(\mathfrak{g})$ be the quantum enveloping algebra of $\mathfrak{g}$; this is an algebra generated by elements $F_{\alpha}, E_{\alpha}, K_{\alpha}^{ \pm 1}$ for $\alpha \in \Pi$, with the relations given in [Jang6, Definition 4.3]. We denote by $U_{q}^{+}(\mathfrak{g}), U_{q}^{-}(\mathfrak{g})$ the subalgebras generated by the $E_{\alpha}$ 's and the $F_{\alpha}$ 's, which are respectively called the positive and negative parts of $U_{q}(\mathfrak{g})$ [Jang6, 4.4]. As shown in [Jan96, Proposition 4.11], $U_{q}(\mathfrak{g})$ is a Hopf algebra.

If $q$ is not a root of unity and chark $>3$ then by [Jan96, chapter 5] for each $\lambda \in$ $\Lambda^{+}$there is an irreducible highest-weight representation of $U_{q}(\mathfrak{g})$ of type 1 , which we denote by $V_{q}(\lambda)$. Each $V_{q}(\lambda)$ decomposes as the direct sum of weight spaces $\bigoplus_{\mu \in \Lambda} V_{q}(\lambda)_{\mu}$; the dimensions of the weight spaces are the same as the corresponding representation over $\mathfrak{g}$, so the Weyl character formula holds for these representations, see [Jan96, 5.15].

4.3. Let $G$ be the simply connected, connected algebraic group with Lie algebra $\mathfrak{g}$. Since $U_{q}(\mathfrak{g})$ is a Hopf algebra, its dual $U_{q}(\mathfrak{g})^{*}$ is an algebra with convolution product induced by the coproduct of $U_{q}(\mathfrak{g})$. There is a map $V_{q}(\lambda)^{*} \otimes V_{q}(\lambda) \longrightarrow U_{q}(\mathfrak{g})^{*}$ defined by sending $\varphi \otimes v \in V_{q}(\lambda)^{*} \otimes V_{q}(\lambda)$ to the linear functional $c_{\varphi, v}^{\lambda}$, which assigns to each $u \in U_{q}(\mathfrak{g})$ the scalar $c_{\varphi, v}^{\lambda}(u)=\varphi(u v)$. Functionals of the form $c_{\varphi, v}^{\lambda}$ are called matrix coefficients. The $\mathbb{k}$-linear span of the matrix coefficients is a subalgebra of $U_{q}(\mathfrak{g})^{*}$ denoted by $\mathcal{O}_{q}[G]$, called the quantized algebra of coordinate functions over the group G [Jan96, 7.11].

4.4. Quantum analogues of flag varieties and their Schubert subvarieties were introduced by Soibelman in [Solg2] and by Lakshmibai and Reshetikhin in [LR92]; we review their definition. We assume that $q$ is not a root of unity. Fix a maximal Borel subgroup $B$ of $G$. The full flag variety associated to $G$ is $G / B$. Let $C_{q}^{+}(\lambda)$ be the vector space of matrix coefficients of the form $c_{\varphi, v_{\lambda}}^{\lambda}$ in $U_{q}(\mathfrak{g})^{*}$, where $v_{\lambda}$ is a highest weight vector in $V_{q}(\lambda)$, and set

$$
\mathcal{O}_{q}[G / B]=\bigoplus_{\lambda \in \Lambda^{+}} C_{q}^{+}(\lambda) \subset \mathcal{O}_{q}[G] \subset U_{q}(\mathfrak{g})^{*}
$$

This is called the quantum full flag variety of $G$. The product of two matrix coefficients in $\mathcal{O}_{q}[G / B]$ is again in $\mathcal{O}_{q}[G / B]$, and its decomposition as a direct sum gives $\mathcal{O}_{q}[G / B]$ the structure of a $\Lambda^{+}$-graded algebra.

Let $I$ be a subset of the set of fundamental weights and set $\mathcal{J}(I)=\sum_{\omega \notin I} \mathbb{N} \omega$. Denote by $W_{I} \subset W$ the subgroup generated by the reflections $s_{\alpha}$ with $\omega_{\alpha} \in I$, and for each class in $W / W_{I}$ pick a representative of minimal length. We denote by $W^{I}$ the set of these representatives. Since the Weyl character formula holds, for each $w \in W$ and 
each $\lambda \in \mathcal{J}(I)$ the vector space $V_{q}(\lambda)_{w \lambda}$ has dimension 1 . The Demazure module $V_{q}(\lambda)_{w}$ is the $U_{q}^{+}(\mathfrak{g})$-submodule of $V_{q}(\lambda)$ generated by a vector of weight $w \lambda$ in $V_{q}(\lambda)$.

The set $I$ determines a Lie subalgebra $\mathfrak{p} \subset \mathfrak{g}$, and a parabolic subgroup $P_{I} \subset G$. The variety $G / P_{I}$ is the corresponding generalized flag variety. To these data we associate the $\Lambda^{+}$-graded subalgebra of $\mathcal{O}_{q}[G / B]$

$$
\mathcal{O}_{q}\left[G / P_{I}\right]=\bigoplus_{\lambda \in \mathcal{J}(I)} C_{q}^{+}(\lambda)
$$

called the quantum partial flag variety associated to $I$.

Given vector spaces $V_{2} \subset V_{1}$, we denote by $V_{2}^{\perp}$ the set of linear functionals over $V_{1}$ which are zero on $V_{2}$. For every $w \in W^{I}$ the vector space

$$
J_{w}^{I}=\bigoplus_{\lambda \in \mathcal{J}(I)}\left\langle c_{\varphi, v_{\lambda}}^{\lambda} \in C_{q}^{+}(\lambda) \mid \varphi \in V_{q}(\lambda)_{w}^{\perp}\right\rangle \subset \mathcal{O}_{q}\left[G / P_{I}\right]
$$

is an ideal of $\mathcal{O}_{q}\left[G / P_{I}\right]$ called the Schubert ideal associated to $w$. The quotient algebra $\mathcal{O}_{q}\left[G / P_{I}\right]_{w}=\mathcal{O}_{q}\left[G / P_{I}\right] / J_{w}^{I}$ is called the quantum Schubert variety associated to $w$.

\section{Degeneration of quantum Schubert varieties}

Our aim is to show that quantum Schubert varieties have quantum affine toric degenerations. In order to do so we work for a moment over the field $\mathbb{Q}(v)$, where $v$ is an indeterminate over $\mathbb{Q}$, and consider the $\mathbb{Q}(v)$-algebra $U=U_{v}(\mathfrak{g})$. We now review Caldero's proof of the existence of an $\left(S,<_{\text {lex }}\right)$-basis of $\mathcal{O}_{v}[G / B]$, and its natural extension to arbitrary partial flag and Schubert varieties [Calo2]. Since Caldero is interested in classical flag varieties, he works with a large base field $\mathbb{C}(q)$ that allows him to specialize at $q=1$ and still get algebraic varieties over the complex numbers. We give a different version of his argument which works over $\mathbb{Q}(v)$.

4.5. Fix as our base field $\mathbb{Q}(v)$, and set $U=U_{v}(\mathfrak{g})$. We denote by $U^{+}$and $U^{-}$the algebras $U_{v}^{+}(\mathfrak{g})$ and $U_{v}^{-}(\mathfrak{g})$, respectively.

Let $\mathcal{A}=\mathbb{Z}\left[v, v^{-1}\right] \subset \mathbb{Q}(v)$. For each $n \in \mathbb{N}$ and each $\alpha \in \Pi$ we write $v_{\alpha}$ for $v^{(\alpha, \alpha) / 2}$. We also use the notation $[n]_{\alpha}=\frac{v_{\alpha}^{n}-v_{\alpha}^{-n}}{v_{\alpha}-v_{\alpha}^{-1}}$, and $[n]_{\alpha} !=[1]_{\alpha}[2]_{\alpha} \cdots[n]_{\alpha}$. Finally, we set $F_{\alpha}^{(n)}=\frac{1}{[n]_{\alpha} !} F_{\alpha}^{n}$ and $E_{\alpha}^{(n)}=\frac{1}{[n]_{\alpha} !} E_{\alpha}^{n}$. The algebra $U$ has an $\mathcal{A}$-form which we denote by $U_{\mathcal{A}}$; it is the $\mathcal{A}$-subalgebra of $U$ generated by the elements of the form $F_{\alpha}^{(n)}, E_{\alpha}^{(n)}, K_{\alpha}^{ \pm 1}$ for all $\alpha \in \Pi$ and all $n \geq 0$ [Jang6, 11.1]. The algebra $U^{+}$, resp. $U^{-}$, also has an $\mathcal{A}$-form which we denote by $U_{\mathcal{A}}^{+}$, resp. $U_{\mathcal{A}}^{-}$; it is generated by all the $E_{\alpha}^{(n)}$, resp. $F_{\alpha}^{(n)}$, with $n \geq 0$. These $\mathcal{A}$-forms are compatible with the weight decomposition of $U$. By construction $U \cong \mathbb{Q}(v) \otimes_{\mathcal{A}} U_{\mathcal{A}}$, and analogous results hold for $U_{\mathcal{A}}^{+}$and $U_{\mathcal{A}}^{-}$.

For the rest of this section we fix a nonzero highest weight vector $v_{\lambda} \in V_{v}(\lambda)$. Setting $V_{\mathcal{A}}(\lambda)=U_{\mathcal{A}}^{-} v_{\lambda} \subset V_{v}(\lambda)$ we obtain $\mathcal{A}$-forms of $U$-modules. These $\mathcal{A}$-forms are compatible with the weight decompositions of the original objects, see [Jang6, chapter 11]. Now let $\lambda$ be a dominant integral weight and fix $w \in W$. The $\mathcal{A}$-form of the Demazure module $V_{v}(\lambda)_{w}$ is defined as $V_{\mathcal{A}}(\lambda)_{w}=U_{\mathcal{A}}^{+} V_{\mathcal{A}}(\lambda)_{w \lambda}$. 
4.6. The algebra $U_{\mathcal{A}}^{-}$has a homogeneous $\mathcal{A}$-basis, called the canonical or global basis of $U^{-}$, discovered independently by Lusztig and Kashiwara. Its construction is the subject of [Jang6, chapters 9 - 11], and we will use the notation from this source to recapitulate some relevant facts.

Set $A_{0} \subset \mathbb{Q}(v)$ to be the ring of rational functions without a pole at 0 . For each $\alpha \in \Pi$ define the operators $\tilde{E}_{\alpha}, \tilde{F}_{\alpha}: U^{-} \longrightarrow U^{-}$as in [Jan96, 10.2], and let $\mathcal{L}(\infty)$ be the $A_{0}$-lattice generated by all elements of the form $\tilde{F}_{\alpha_{1}} \tilde{F}_{\alpha_{2}} \cdots \tilde{F}_{\alpha_{r}}(1)$; by definition these are weight elements, so setting $\mathcal{L}(\infty)_{-v}=\mathcal{L}(\infty) \cap U_{-v}^{-}$for each $v \in \mathbb{Z} \Phi$ with $v \geq 0$, we get $\mathcal{L}(\infty)=\bigoplus_{v>0} \mathcal{L}(\infty)_{-v}$, and furthermore each $\mathcal{L}(\infty)_{-v}$ is a finitely generated $A_{0}$-module that generates $U_{v}^{-}$over $\mathbb{Q}(v)$.

Set

$$
\mathcal{B}(\infty)_{-v}=\left\{\tilde{F}_{\alpha_{1}} \tilde{F}_{\alpha_{2}} \cdots \tilde{F}_{\alpha_{r}}(1)+v \mathcal{L}(\infty) \mid \sum_{i} \alpha_{i}=v\right\} \subset \mathcal{L}(\infty)_{-v} / v \mathcal{L}(\infty)_{-v},
$$

and set $\mathcal{B}(\infty)=\bigsqcup_{v \geq 0} \mathcal{B}(\infty)_{-v}$. Although it is not obvious, $\mathcal{B}(\infty)_{-v}$ is a basis of $\mathcal{L}(\infty)_{-v} / v \mathcal{L}(\infty)_{-v}$ [Jan96, Proposition 10.11]; this is the crystal basis of $U_{-v}^{-}$at $v=0$. It turns out that each $b \in \mathcal{B}(\infty)_{-v}$ has a unique lift $G(b) \in \mathcal{L}(\infty)_{-v} \cap U_{\mathcal{A}^{-}}^{-}$, which is invariant under the action of certain automorphisms of $U$ [Jang6, Theorem 11.10 a)]. The set $G(\mathcal{B})$ of all $G(b)$ with $b \in \mathcal{B}(\infty)$ is the global basis of $U_{\mathcal{A}}^{-}$.

Let $w \in W$, and let $w=s_{\alpha_{1}} \cdots s_{\alpha_{r}}$ be a reduced decomposition of $w$. Set $\mathcal{B}_{w}(\infty)$ as the set of elements in $\mathcal{B}(\infty)$ of the form $\tilde{F}_{\alpha_{1}}^{k_{1}} \tilde{F}_{\alpha_{2}}^{k_{2}} \cdots \tilde{F}_{\alpha_{r}}^{k_{r}}(1)+v \mathcal{L}(\infty)$ with $k_{j} \geq 0$. This set does not depend on the decomposition of $w$ [Kas93, Proposition 3.2.5].

Theorem. Let $v \in \mathbb{Z} \Phi$ with $v \geq 0$.

(a) The set $\left\{G(b) \mid b \in \mathcal{B}(\infty)_{-v}\right\}$ is an $\mathcal{A}$-basis of $\left(U_{\mathcal{A}}^{-}\right)_{-v}$.

(b) Let $\lambda$ be a dominant integral weight. The set $\left\{G(b) v_{\lambda} \mid b \in \mathcal{B}(\infty)_{-v}\right\} \backslash\{0\}$ is an $\mathcal{A}$-basis of $V_{\mathcal{A}}(\lambda)_{\lambda-v}$. Furthermore, if $G(b) v_{\lambda}=G\left(b^{\prime}\right) v_{\lambda} \neq 0$ then $b=b^{\prime}$.

(c) Let $w \in W$. The set $B_{w}(\lambda)=\left\{G(b) v_{\lambda} \mid b \in \mathcal{B}_{w}(\infty)\right\} \backslash\{0\}$ is an $\mathcal{A}$-basis of $V_{\mathcal{A}}(\lambda)_{w}$.

Proof. The first two items are part of [Jan96, Theorem 11.10]. The third is [Kas93. Proposition 3.2.5 (vi)].

4.7. We now use the global basis to produce bases for quantum Schubert varieties. For each dominant integral weight $\lambda$ we write $\mathcal{B}(\lambda)=\left\{b \in \mathcal{B}(\infty) \mid G(b) v_{\lambda} \neq 0\right\}$. By the previous theorem the set $\left\{G(b) v_{\lambda} \mid b \in \mathcal{B}(\lambda)\right\}$ is a basis of $V_{v}(\lambda)$, so we can take its dual basis. Given $b \in \mathcal{B}(\lambda)$, we denote by $b_{\lambda}^{*}$ the unique element of $V_{v}(\lambda)^{*}$ such that $b_{\lambda}^{*}\left(G\left(b^{\prime}\right) v_{\lambda}\right)=\delta_{b, b^{\prime}}$ for all $b^{\prime} \in \mathcal{B}(\lambda)$. Thus to each element $b \in \mathcal{B}(\lambda)$ we can associate the matrix coefficient $b_{\lambda}=c_{b_{,}^{*}, v_{\lambda}}^{\lambda} \in C_{v}^{+}(\lambda)$, and the set $\left\{b_{\lambda} \mid b \in \mathcal{B}(\lambda)\right\}$ is a basis of $C_{v}^{+}(\lambda)$. Since the quantum flag variety is the direct sum of all these spaces with $\lambda$ running over all dominant integral weights, we obtain a basis of $\mathcal{O}_{v}[G / B]$ as defined in 4.4 by taking

$$
F B=\left\{b_{\lambda} \mid b \in \mathcal{B}(\lambda), \lambda \in \Lambda^{+}\right\} .
$$


If $I$ is a subset of the fundamental weights, then we obtain a basis of the partial flag variety $\mathcal{O}_{v}\left[G / P_{I}\right]$ by taking

$$
F B_{I}=\left\{b_{\lambda} \mid b \in \mathcal{B}(\lambda), \lambda \in \mathcal{J}(I)\right\} .
$$

Finally, the third item of the previous theorem implies that the ideal $J_{w}^{I}$ defined in $4 \cdot 4$ is spanned over $\mathbb{Q}(v)$ by all elements of the form $b_{\lambda}$ with $b \in \mathcal{B}(\lambda) \backslash \mathcal{B}_{w}(\infty)$. Setting $\mathcal{B}_{w}(\lambda)=\mathcal{B}_{w}(\infty) \cap \mathcal{B}(\lambda)$ we obtain a basis for the quantum Schubert variety $\mathcal{O}_{v}\left[G / P_{I}\right]_{w}$ by taking the image of

$$
S B_{I}(w)=\left\{b_{\lambda} \mid b \in \mathcal{B}_{w}(\lambda), \lambda \in \mathcal{J}(I)\right\} .
$$

in the quotient.

4.8. Recall that the Kashiwara operators $\tilde{E}_{\alpha}, \tilde{F}_{\alpha}$ induce operators $\tilde{E}_{\alpha}, \tilde{F}_{\alpha}: \mathcal{B}(\infty) \longrightarrow$ $\mathcal{B}(\infty) \cup\{0\}$ [Jang6, 10.12]. By definition the operators $\tilde{E}_{\alpha}$ are locally nilpotent as operators on $U[$ [Jang6, 10.2] and hence as operators on $\mathcal{B}(\infty)$, so it makes sense to set $e_{\alpha}(b)=\max \left\{k \in \mathbb{N} \mid \tilde{E}_{\alpha}^{k}(b) \neq 0\right\}$ for each $b \in \mathcal{B}(\infty)$. We write $\bar{E}_{\alpha}(b)=\tilde{E}_{\alpha}^{e_{\alpha}(b)}(b)$.

To each reduced decomposition of $w \in W$ we can associate a parametrization of $\mathcal{B}_{w}(\infty)$, known as a string parametrization, introduced by Littelmann [Lit98] and Berenstein and Zelevinsky [BZ01]. If $\tilde{w}=s_{\alpha_{1}} \cdots s_{\alpha_{r}}$ is the chosen decomposition then we define $\Lambda_{\tilde{w}}: \mathcal{B}(\infty) \longrightarrow \mathbb{N}^{r}$ by the formula

$$
\Lambda_{\tilde{w}}(b)=\left(e_{\alpha_{1}}(b), e_{\alpha_{2}}\left(\bar{E}_{\alpha_{1}}(b)\right), \ldots, e_{\alpha_{r}}\left(\bar{E}_{\alpha_{r-1}} \ldots \bar{E}_{\alpha_{1}}(b)\right)\right) .
$$

If $a=\Lambda_{\tilde{b}}$ then $\tilde{F}_{\alpha_{1}}^{a_{1}} \cdots F_{\alpha_{N}}^{a_{N}} 1=b$, so this map is injective. Now according to [BZo1, Proposition 3.5], the set $S_{\tilde{w}}=\Lambda_{\tilde{w}}\left(\mathcal{B}_{w}(\infty)\right)$ is the set of integral points of a convex polyhedral cone, and hence by Gordan's lemma a normal affine semigroup.

A decomposition of $w_{0}$, the longest word of $W$, is said to be adapted to $w$ if it is of the form $s_{\alpha_{1}} \cdots s_{\alpha_{N}}$ with $s_{\alpha_{1}} \cdots s_{\alpha_{\ell(w)}}=w$. For every element $w \in W$ there exists a decomposition of the longest word of $W$ adapted to $w$, or in other words the longest word of $W$ is the maximum for the weak right Bruhat order on $W$, see [BBo5. Proposition 3.1.2].

Definition. Set $\Pi=\left\{\alpha_{1}, \ldots, \alpha_{n}\right\}$ and set $\omega_{i}=\omega_{\alpha_{i}}$. Fix $w \in W$ and fix $\tilde{w}_{0}$ a decomposition of $w_{0}$ adapted to $w$. We define

$$
\begin{aligned}
\Gamma_{\tilde{w}_{0}}: F B & \longrightarrow \mathbb{N}^{N} \times \mathbb{N}^{n} \\
b_{\lambda} & \longmapsto \Lambda_{\tilde{w}_{0}}(b) \times\left(\left\langle\lambda, \alpha_{1}\right\rangle, \ldots,\left\langle\lambda, \alpha_{n}\right\rangle\right) .
\end{aligned}
$$

and set $\tilde{S}_{\tilde{w}_{0}}=\Gamma_{\tilde{w}_{0}}(F B), \tilde{S}_{\tilde{w}_{0}}^{I}=\Gamma_{\tilde{w}_{0}}\left(F B_{I}\right)$ and $\tilde{S}_{\tilde{w}_{0}, \tilde{w}}^{I}=\Gamma_{\tilde{w}_{0}}\left(S B_{I}(w)\right)$.

If $\Gamma_{\tilde{w}_{0}}\left(b_{\lambda}\right)=\Gamma_{\tilde{w}_{0}}\left(b_{\lambda^{\prime}}^{\prime}\right)$ then $\lambda=\lambda^{\prime}$ and $\Lambda_{\tilde{w}_{0}}\left(b_{\lambda}\right)=\Lambda_{\tilde{w}_{0}}\left(b_{\lambda^{\prime}}^{\prime}\right)$, which implies $b_{\lambda}=b_{\lambda^{\prime}}^{\prime}$. Thus $\Gamma_{\tilde{w}_{0}}$ is injective and the sets $\tilde{S}_{\tilde{w}_{0}}, \tilde{S}_{\tilde{w}_{0}}^{I}$ and $\tilde{S}_{\tilde{w}_{0}, \tilde{w}}^{I}$ parametrize bases of the quantized coordinate rings of the full flag variety, the partial flag variety associated to the set $I$, and of the Schubert variety associated to $I$ and $w$, respectively.

Lemma. The sets $\tilde{S}_{\tilde{w}_{0}}, \tilde{S}_{\tilde{w}_{0}}^{I}$ and $\tilde{S}_{\tilde{w}_{0}, \tilde{w}}^{I}$ are normal affine semigroups. 
Proof. According to [Lit98, Proposition 1.5] $\tilde{S}_{\tilde{w}_{0}}$ is the set of all $a \times\left(r_{1}, \ldots, r_{n}\right) \in S_{\tilde{w}_{0}} \times$ $\mathbb{N}^{n}$ such that

$$
a_{l} \leq\left\langle\lambda-\sum_{j=1}^{N-l} a_{N-j+1} \alpha_{i_{N-j+1}}, \alpha_{i_{l}}\right\rangle \quad(1 \leq l \leq N)
$$

where $\lambda=\sum_{i} r_{i} \omega_{i}$. Thus $\tilde{S}_{\tilde{w}_{0}}$ is the set of points of $S_{\tilde{w}_{0}} \times \mathbb{N}^{n}$ that comply with these inequalities, and hence it is also a normal affine semigroup.

Furthermore, $\tilde{S}_{\tilde{w}_{0}}^{I}=\Gamma_{\tilde{w}_{0}}\left(F B_{I}\right)$ is the intersection of $\tilde{S}_{\tilde{w}_{0}}$ with the hyperplanes defined by the equations $x_{N+i}=0$ for all $i$ such that $\omega_{i} \in I$, and hence is also a normal affine semigroup. Finally, the fact that the decomposition $\tilde{w}_{0}$ is adapted to $w$ implies that $\tilde{S}_{\tilde{w}_{0}, \tilde{w}}^{I}=\Gamma_{\tilde{w}_{0}}\left(S B_{I}(w)\right)$ is the intersection of $\tilde{S}_{\tilde{w}_{0}}^{I}$ with the hyperplanes defined by $x_{i}=0$ for all $\ell(w)<i \leq N$, and hence it is also a normal affine semigroup.

4.9. We have just shown that the bases of quantum flag varieties and quantum Schubert varieties defined in 4.7 are parametrized by normal semigroups. All that is left to check is that they have the multiplicative property of $\left(S,<_{\text {lex }}\right)$-bases, where $<_{\text {lex }}$ is the lexicographic order of $\mathbb{N}^{N} \times \mathbb{N}^{n}$.

Let $\lambda, \lambda^{\prime} \in \Lambda^{+}$and $b \in \mathcal{B}(\lambda), b^{\prime} \in \mathcal{B}\left(\lambda^{\prime}\right)$. Recall that $b_{\lambda}^{*}$ denotes the element in the dual basis of $V_{v}(\lambda)^{*}$ as defined in 4.7. The product $b_{\lambda} b_{\lambda^{\prime}}^{\prime}$ is by definition the matrix coefficient corresponding to the functional $b_{\lambda}^{*} \otimes b_{\lambda^{\prime}}^{*}$ and the vector $v_{\lambda} \otimes v_{\lambda^{\prime}}$ over $V(\lambda) \otimes V\left(\lambda^{\prime}\right)$. Now the $U$-module generated by $v_{\lambda} \otimes v_{\lambda^{\prime}}$ is isomorphic to $V_{v}\left(\lambda+\lambda^{\prime}\right)$, so $b_{\lambda}^{*} \otimes b_{\lambda^{\prime}}^{*}$ naturally induces an element in $C_{v}^{+}\left(\lambda+\lambda^{\prime}\right)$, and the product $b_{\lambda} b_{\lambda^{\prime}}^{\prime}$ is a linear combination of matrix coefficients in $C_{v}^{+}\left(\lambda+\lambda^{\prime}\right)$

$$
b_{\lambda} b_{\lambda^{\prime}}^{\prime}=\sum_{b^{\prime \prime} \in \mathcal{B}\left(\lambda+\lambda^{\prime}\right)} c_{b, b^{\prime}}^{b^{\prime \prime}} b_{\lambda+\lambda^{\prime}}^{\prime \prime}
$$

with $c_{b, b^{\prime}}^{b^{\prime \prime}} \in \mathbb{Q}(v)$. In order to show that $F B$ is an $\left(\tilde{S}_{\tilde{\omega}_{0}},<\right.$ lex $)$-basis we must show that $c_{b, b^{\prime}}^{b^{\prime \prime}} \neq 0$ implies that $\Gamma_{\tilde{\omega}_{0}}\left(b^{\prime \prime}\right) \leq \Gamma_{\tilde{\omega}_{0}}(b)+\Gamma_{\tilde{\omega}_{0}}\left(b^{\prime}\right)$, and that if equality holds then $c_{b, b^{\prime}}^{b^{\prime \prime}}$ is nonzero. Notice that this would also imply that $F B_{I}$ is an $\left(\tilde{S}_{\tilde{\omega}_{0}}^{I},<_{\text {lex }}\right)$-basis and that $S B_{I}(\tilde{w})$ is a $\left(\tilde{S}_{\tilde{\omega}_{0}, \tilde{w}}^{I},<_{\text {lex }}\right)$-basis.

By definition of a dual basis the scalar $c_{b, b^{\prime}}^{b^{\prime \prime}}$ is the value $b_{\lambda} b_{\lambda^{\prime}}^{\prime}\left(G\left(b^{\prime \prime}\right)\right)$. On the other hand, by the definition of the product of matrix coefficients

$$
b_{\lambda} b_{\lambda^{\prime}}^{\prime}\left(G\left(b^{\prime \prime}\right)\right)=b_{\lambda}^{*} \otimes b_{\lambda^{\prime}}^{\prime *}\left(\Delta\left(G\left(b^{\prime \prime}\right)\right) \cdot v_{\lambda} \otimes v_{\lambda^{\prime}}\right),
$$

so we need to study the coproduct $\Delta\left(G\left(b^{\prime \prime}\right)\right)$. It follows from [Jang6, 4.9 (4)] that $\Delta\left(F_{\alpha}^{(r)}\right)=\sum_{i+j=r} v_{\alpha}^{j i} F_{\alpha}^{(i)} \otimes F_{\alpha}^{(j)} K_{\alpha}^{-i}$, where $v_{\alpha}=v^{(\alpha, \alpha) / 2}$. Thus by an argument similar to that of [Jan96, Lemma 4.12] we see that

$$
\Delta\left(U_{\mathcal{A}}^{-}\right)_{-v} \subset \bigoplus_{0 \leq \mu \leq \nu}\left(U_{\mathcal{A}}^{-}\right)_{-\mu} \otimes\left(U_{\mathcal{A}}^{-}\right)_{-(v-\mu)} K_{-\mu}
$$

It follows that $\Delta\left(G\left(b^{\prime \prime}\right)\right)$ is an $\mathcal{A}$-linear combination of terms $G\left(b_{(1)}\right) \otimes G\left(b_{(2)}\right) K_{\mu}$, with $b_{(1)}, b_{(2)} \in \mathcal{B}(\infty)$, and $\mu$ the weight of $b_{(1)}$. Among all these terms there is one of the 
form $d_{b, b^{\prime}}^{b^{\prime \prime}}\left(G(b) \otimes G\left(b^{\prime}\right) K_{\mathrm{wt}(b)}\right)$ with $d_{b, b^{\prime}}^{b^{\prime \prime}} \in \mathcal{A}$, and thus $c_{b, b^{\prime}}^{b^{\prime \prime}}=v^{\left(\mathrm{wt}(b), \lambda^{\prime}\right)} d_{b, b^{b^{\prime}}}^{b^{\prime \prime}}$. Notice that unlike before, the element $d_{b, b^{\prime}}^{b^{\prime \prime}}$ is independent of $\lambda$ and $\lambda^{\prime}$.

The problem of showing that $F B$ is indeed an $\left(\tilde{S}_{\tilde{w}_{0}},<_{\text {lex }}\right)$-basis thus reduces to showing that if $d_{b, b^{\prime}}^{b^{\prime \prime}} \neq 0$ then $\Lambda_{\tilde{w}_{0}}\left(b^{\prime \prime}\right) \leq_{\text {lex }} \Lambda_{\tilde{w}_{0}}(b)+\Lambda_{\tilde{w}_{0}}\left(b^{\prime}\right)$; this also implies that $F B_{I}$ and $S B_{I}(\tilde{w})$ are $\left(\tilde{S}_{\tilde{w}_{0}},<\right.$ lex $)$-bases. Caldero shows that this is indeed the case in [Calo2, Theorem 2.3], under the hypothesis that $q$ is transcendental over $\mathbb{C}$. We give an alternative proof in the following paragraphs.

4.10. We fix some notation. Given a decomposition of the longest word of $W \tilde{w}_{0}=$ $s_{\alpha_{1}} \cdots s_{\alpha_{N}}$, for each $a \in \mathbb{N}^{N}$ we write $F^{(a)}=F_{\alpha_{1}}^{\left(a_{1}\right)} \cdots F_{\alpha_{N}}^{\left(a_{N}\right)}$ and $\tilde{F}^{a}=\tilde{F}_{\alpha_{1}}^{a_{1}} \cdots \tilde{F}_{\alpha_{N}}^{a_{N}}$. If $a$ lies in the image of $\Lambda_{\tilde{w}_{0}}$ then we write $b^{a}=\Lambda_{\tilde{w}_{0}}^{-1}(a)$.

According to [Lit98, Proposition 10.3] the monomials $F^{(a)}$ with $a$ in the image of $\Lambda_{\tilde{\omega}_{0}}$ form a weight basis of $U_{\mathcal{A}}^{-}$. In fact, if we fix $v \in \mathbb{Z} \Phi, v \geq 0$, the change of basis matrix between Littelman's monomial basis and the global basis of $\left(U_{\mathcal{A}}^{-}\right)_{-v}$ is unipotent if we order the bases according to the lexicographic order of the corresponding $a \in \operatorname{Im} \Lambda_{\tilde{\omega}_{0}}$. The following lemma records this fact. On the other hand, we can consider a monomial of the form $F^{(a)}$ with $a$ outside the image of $\Lambda_{\tilde{\omega}_{0}}$; we show that in this case the monomial is a linear combination of monomials whose exponents are strictly larger than $a$ in the lexicographic order, or equivalently, elements $G\left(b^{a^{\prime}}\right)$ with $a^{\prime}>_{\operatorname{lex}} a$.

Lemma. Fix $\tilde{w}_{0}=s_{\alpha_{1}} \cdots s_{\alpha_{N}}$ a decomposition of the longest word of $W$. Let $a \in \mathbb{N}^{N}$, and let $v \in \mathbb{Z} \Phi, v \geq 0$ be such that $F^{(a)} \in U_{-v}^{-}$.

(a) Let $\alpha \in \Pi$ and $r>0$. Let $b \in \mathcal{B}(\infty)_{-v}$. Then for each $b^{\prime} \in \mathcal{B}(\infty)_{-v-r \alpha}$ with $e_{\alpha}\left(b^{\prime}\right)>r+e_{\alpha}(b)$ there exists $x_{b^{\prime}} \in \mathcal{A}$ such that

$$
F_{\alpha}^{(r)} G(b)=\left[\begin{array}{c}
r+e_{\alpha}(b) \\
r
\end{array}\right] G\left(\tilde{F}_{\alpha}^{r} b\right)+\sum_{e_{\alpha}\left(b^{\prime}\right)>r+e_{\alpha}(b)} x_{b^{\prime}} G\left(b^{\prime}\right) .
$$

(b) Suppose a lies in the image of $\Lambda_{\tilde{w}_{0}}$. Then for each $a^{\prime} \in \Lambda_{\tilde{w}_{0}}\left(\mathcal{B}(\infty)_{-v}\right)$ with $a^{\prime}>_{\text {lex }} a$ there exist $x_{a, a^{\prime}}, y_{a, a^{\prime}} \in \mathcal{A}$ such that

$$
F^{(a)}=G\left(b^{a}\right)+\sum_{a^{\prime}>\operatorname{lex} a} x_{a, a^{\prime}} G\left(b^{a^{\prime}}\right), \quad G\left(b^{a}\right)=F^{(a)}+\sum_{a^{\prime}>\operatorname{lex} a} y_{a, a^{\prime}} F^{\left(a^{\prime}\right)} .
$$

(c) Suppose a does not lie in the image of $\Lambda_{\tilde{w}_{0}}$. Let $b=\tilde{F}^{a} 1+v \mathcal{L}(\infty) \in \mathcal{B}(\infty)_{-v}$ and let $s=\Lambda_{\tilde{w}_{0}}(b)$. Then $s>_{\text {lex }} a$, and for each $a^{\prime} \in \Lambda_{\tilde{w}_{0}}\left(\mathcal{B}(\infty)_{-v}\right)$ with $a^{\prime}>_{\text {lex }} a$ there exists $z_{a, a^{\prime}} \in \mathcal{A}$ such that

$$
F^{(a)}=\sum_{a^{\prime}>\operatorname{lex} a} z_{a, a^{\prime}} G\left(b^{a^{\prime}}\right) .
$$

Furthermore $z_{a, s} \neq 0$. 
Proof. Recall from [Jan96, Lemma 11.3] that $\tilde{F}_{\alpha}^{r}\left(U_{\mathcal{A}}^{-}\right)_{-v}=\sum_{s \geq 0} F_{\alpha}^{(r+s)}\left(U_{\mathcal{A}}^{-}\right)_{-v+s \alpha}$, and that for each $u \in\left(U_{\mathcal{A}}^{-}\right)_{-v}$ we have

$$
\tilde{F}_{\alpha}^{r} u=F_{\alpha}^{(r)} u+\tilde{F}_{\alpha}^{r+1} u^{\prime}=F_{\alpha}^{(r)} u+\sum_{s \geq 1} F_{\alpha}^{(r+s)} u_{s}
$$

where $u^{\prime} \in\left(U_{\mathcal{A}}^{-}\right)_{v+\alpha}$ and $u_{s} \in\left(U_{\mathcal{A}}^{-}\right)_{v+s \alpha}$. Set $e=e_{\alpha}(b)$. As shown in the proof of [Jan96, Lemma 11.12], in p. 249 below equation (1),

$$
G(b)=\tilde{F}_{\alpha}^{e}\left(G\left(\tilde{E}_{\alpha}^{e} b\right)\right)+\tilde{F}_{\alpha}^{e+1} u^{\prime}
$$

with $u^{\prime} \in\left(U_{\mathcal{A}}^{-}\right)_{v+\alpha}$, so

$$
G(b)=F_{\alpha}^{(e)}\left(G\left(\tilde{E}_{\alpha}^{e} b\right)\right)+\sum_{s \geq 1} F_{\alpha}^{(e+s)} u_{s}
$$

Multiplying this by $F_{\alpha}^{(r)}$ we get

$$
F_{\alpha}^{(r)} G(b)=\left[\begin{array}{c}
r+e \\
r
\end{array}\right] F_{\alpha}^{(r+e)}\left(G\left(\tilde{E}_{\alpha}^{e} b\right)\right)+\sum_{s \geq 1} F_{\alpha}^{(r+e+s)} u_{s}^{\prime} .
$$

Using $⿴$ we get

$$
\tilde{F}_{\alpha}^{r+e}\left(G\left(\tilde{E}_{\alpha}^{e} b\right)\right)=\tilde{F}_{\alpha}^{r+e}\left(G\left(\tilde{E}_{\alpha}^{e+r} \tilde{F}_{\alpha}^{r} b\right)\right)=G\left(\tilde{F}_{\alpha}^{r} b\right)+\tilde{F}_{\alpha}^{r+e+1} u^{\prime \prime}
$$

and finally

$$
F_{\alpha}^{(r)} G(b) \equiv\left[\begin{array}{c}
r+e \\
r
\end{array}\right] G\left(\tilde{F}_{\alpha}^{r} b\right) \bmod \tilde{F}_{\alpha}^{r+e+1}\left(U_{\mathcal{A}}^{-}\right)_{-v}
$$

By [Jan96, Lemma 11.12] the $G\left(b^{\prime}\right)$ with $e_{\alpha}\left(b^{\prime}\right)>r+e$ form an $\mathcal{A}$-basis of $\tilde{F}_{\alpha}^{r+e+1}\left(U_{\mathcal{A}}^{-}\right)_{-v}$, so we are done with item (a).

As mentioned above, item (b) is a consequence of [Lit98. Proposition 10.3], which states that given a dominant integral weight $\lambda$ and a highest weight vector $v_{\lambda} \in V_{\mathcal{A}}(\lambda)$, then if $G\left(b^{a}\right) v_{\lambda} \neq 0$ there exist $x_{a, a^{\prime}}$ as in the statement such that

$$
F^{(a)} v_{\lambda}=G\left(b^{a}\right) v_{\lambda}+\sum_{a^{\prime}>\operatorname{lex} a} x_{a, a^{\prime}} G\left(b^{a^{\prime}}\right) v_{\lambda}
$$

As shown in the proof of [Jan96. Theorem 10.10], there exists a dominant integral weight $\lambda$ such that the map $U_{-v}^{-} \longrightarrow V_{v}(\lambda)_{\lambda-v}$ given by $G(b) \mapsto G(b) v_{\lambda}$ is an isomorphism, so the first formula is proved. This means that the (finite) matrix of the coefficients of the $F^{(a)} \in\left(U_{\mathcal{A}}^{-}\right)_{-v}$ in the global basis of $\left(U_{\mathcal{A}}^{-}\right)_{-v}$ with the order induced by the lexicographic order, is lower triangular with ones in the diagonal. The second formula follows by taking the inverse of this matrix. 
Let us prove the last item. Suppose that $s<\operatorname{lex} a$. Then by definition $s_{1}=$ $a_{1}, \ldots, s_{j}=a_{j}, s_{j+1}<a_{j+1}$ for some $1 \leq j \leq N$. This implies that

$$
\tilde{E}_{\alpha_{j+1}}^{s_{j+1}+1} \tilde{E}_{\alpha_{j}}^{s_{j}} \cdots \tilde{E}_{\alpha_{1}}^{s_{1}}(b)=\tilde{F}_{\alpha_{j+1}}^{a_{j}-s_{j}-1} \cdots \tilde{F}_{\alpha_{N}}^{a_{N}} 1+v \mathcal{L}(\infty) \neq 0,
$$

since the maps $\tilde{F}_{\alpha}$ are injective. This contradicts the definition of $\Lambda_{\tilde{w}_{0}}(b)$, so $s \geq \operatorname{lex} a$ and since $a$ is not in the image of $\Lambda_{\tilde{w}_{0}}$ the inequality is strict.

We now prove the following intermediate result: for each $a \in \mathbb{N}^{N}$ we have $F^{(a)}=$ $z_{a, s}^{\prime} G\left(b^{s}\right)+\sum_{a^{\prime}>\operatorname{lex} a} z_{a, a^{\prime}}^{\prime} F^{\left(a^{\prime}\right)}$, where $s=\Lambda_{\tilde{\omega}_{0}}^{-1}\left(\tilde{F}^{a}(1+v \mathcal{L}(\infty))\right)$ and $z_{a, a^{\prime}}^{\prime}, z_{a, s}^{\prime}$ lie in $\mathcal{A}$ for all $a^{\prime}$. We prove this by descending induction on $j=\min \left\{i \mid a_{i} \neq 0\right\}$, starting with the case $j=N$. In that case item (a) implies that $\tilde{F}^{a}=G\left(b^{a}\right)$ and we are done.

Now let $\bar{a} \in \mathbb{N}^{N}$ be the $N$-tuple given by $\bar{a}_{j}=0$ and $\bar{a}_{i}=a_{i}$ for all $i \neq j$. By the inductive hypothesis we have

$$
F^{(a)}=F_{\alpha_{j}}^{\left(a_{j}\right)} F^{(\bar{a})}=z_{\bar{a}, \bar{s}}^{\prime} F_{\alpha_{j}}^{a_{j}} G\left(b^{\bar{s}}\right)+\sum_{\bar{a}^{\prime}>\bar{a}} z_{\bar{a}, \bar{a}^{\prime}}^{\prime} F_{\alpha_{j}}^{\left(a_{j}\right)} F^{\left(\bar{a}^{\prime}\right)} .
$$

Now $F_{\alpha_{j}}^{\left(a_{j}\right)} F^{\left(\bar{a}^{\prime}\right)}$ is a scalar multiple of $F^{\left(a^{\prime}\right)}$ where $a_{j}^{\prime}=a_{j}$ and $a_{i}^{\prime}=\bar{a}_{i}$, and clearly $a^{\prime}>_{\text {lex }} a$. On the other hand by item (a)

$$
F_{\alpha_{j}}^{a_{j}} G\left(b^{\bar{s}}\right)=\left[\begin{array}{c}
a_{j}+e \\
a_{j}
\end{array}\right] G\left(\tilde{F}_{\alpha_{j}}^{a_{j}} b^{\bar{s}}\right)+\sum_{e_{\alpha_{j}}\left(b^{\prime}\right)>a_{j}+e_{\alpha_{j}}(b)} x_{b^{\prime}} G\left(b^{\prime}\right)
$$

where $e=e_{\alpha_{j}}\left(b^{\bar{s}}\right)$. Since $b^{\bar{s}}=\tilde{F}^{\bar{a}}$ we have $\tilde{F}_{\alpha_{j}}^{a_{j}} b^{s}=\tilde{F}^{a}(1+v \mathcal{L}(\infty))=b^{s}$. On the other hand, the condition $e_{\alpha_{j}}\left(b^{\prime}\right)>a_{j}+e_{\alpha_{j}}(b)$ guarantees that $\Lambda_{\tilde{\omega}_{0}}\left(b^{\prime}\right)>_{\text {lex }} a$ and by item (b) each $G\left(b^{\prime}\right)$ is a linear combination of monomials $F^{\left(a^{\prime}\right)}$ with $a^{\prime}>_{\text {lex }} a$. This completes the proof of the intermediate result.

Now consider the set $D$ of all $a \notin \operatorname{Im} \Lambda_{\tilde{\omega}_{0}}$ such that $F^{(a)} \in\left(U_{\mathcal{A}}^{-}\right)_{-v}$ for which the statement of (c) fails. This set is finite and totally ordered by the lexicographic order, so if it is not empty then it has a maximal element $d$. Now by the intermediate statement $F^{(d)}=z_{d, s}^{\prime} G\left(b^{s}\right)+\sum_{d^{\prime}>\operatorname{lex}} z_{d, d^{\prime}}^{\prime} F^{\left(d^{\prime}\right)}$, and since no $d^{\prime}$ can be in $D$, this sum is an $\mathcal{A}$-linear combination of elements $G\left(b^{s^{\prime}}\right)$ with $s^{\prime}>_{\text {lex }} d^{\prime}>_{\text {lex }} d$. This contradicts the fact that $d \in D$, and the contradiction arose from supposing $D$ was nonempty. Thus (c) holds in all cases.

4.11. Recall that we have defined $c_{b, b^{\prime}}^{b^{\prime \prime}} \in \mathcal{A}$ as the coefficient of $b_{\lambda+\lambda^{\prime}}^{\prime \prime}$ in $b_{\lambda} b_{\lambda^{\prime}}$, and that we have shown that it equals a power of $v$ times $d_{b, b^{\prime}}^{b^{\prime \prime}}$, the coefficient of $G(b) \otimes G\left(b^{\prime}\right) K_{-\mu}$ in $\Delta\left(G\left(b^{\prime \prime}\right)\right)$, where $-\mu$ is the weight of $G(b)$. We have also shown above that the statement of the following proposition is equivalent to $F B$ being a $\left(\tilde{S}_{\tilde{\omega}_{0}},<_{\text {lex }}\right)$-basis.

Proposition. Fix $\tilde{w}_{0}=s_{\alpha_{1}} \cdots s_{\alpha_{N}}$ a decomposition of the longest word of $W$. Let $b, b^{\prime}, b^{\prime \prime} \in$ $\mathcal{B}(\infty)$. If $d_{b, b^{\prime}}^{b^{\prime \prime}} \neq 0$ then $\Lambda_{\tilde{w}_{0}}\left(b^{\prime \prime}\right) \leq$ lex $\Lambda_{\tilde{w}_{0}}(b)+\Lambda_{\tilde{w}_{0}}\left(b^{\prime}\right)$, and if equality holds then $d_{b, b^{\prime}}^{b^{\prime \prime}}$ is a power of $v$. 
Proof. We have already observed that $\Delta\left(F_{\alpha}^{(r)}\right)=\sum_{i+j=r} v_{\alpha}^{i j} F_{\alpha}^{(i)} \otimes F_{\alpha}^{(j)} K_{\alpha}^{-i}$, and it follows that for each $a \in \mathbb{N}^{N}$ we get $\Delta\left(F^{(a)}\right)=\sum_{t+u=a} v^{z(t, u)} F^{(t)} \otimes F^{(u)} K_{\mathrm{wt}\left(F^{(t)}\right)}$, with $z(t, u) \in \mathbb{Z}$. By item (b) of the previous lemma

$$
\begin{aligned}
\Delta\left(G\left(b^{a}\right)\right) & =\Delta\left(F^{(a)}\right)+\sum_{a^{\prime}>\operatorname{lex} a} y_{a, a^{\prime}} \Delta\left(F^{\left(a^{\prime}\right)}\right) \\
& =\sum_{t+u=a} v^{z(t, u)} F^{(t)} \otimes F^{(u)} K_{\mathrm{wt}\left(F^{(t)}\right)}+\sum_{t+u>\operatorname{lex} a} y_{a, t+u} v^{z(t, u)} F^{(t)} \otimes F^{(u)} K_{\mathrm{wt}\left(F^{(t)}\right)}
\end{aligned}
$$

and using item (c) of the lemma we get that the element in the last display equals

$$
\sum_{t+u=a} v^{z(t, u)} G\left(b^{t}\right) \otimes G\left(b^{u}\right) K_{\mathrm{wt}\left(b^{t}\right)}+\sum_{t+u>\operatorname{lex} a} y_{t, u}^{\prime} G\left(b^{t}\right) \otimes G\left(b^{u}\right) K_{\mathrm{wt}\left(b^{t}\right)}
$$

where $t, u$ run over the image of $\Lambda_{\tilde{w}_{0}}$, and $z(t, u) \in \mathbb{Z}$ and $y_{t, u}^{\prime} \in \mathcal{A}$ for each such pair $(t, u)$. The result follows by taking $b^{a}=b^{\prime \prime}$.

Remark. It is possible to take an alternative approach, and define bases for quantum flag and Schubert varieties as in paragraph $4 \cdot 7$ starting with the monomial basis instead of the global basis, and in this case we also obtain an $\left(S,<_{\text {lex }}\right)$-basis. This is the approach taken by Fang, Fourier and Littelmann in [FFL17b], where they obtain many different monomial bases for (classical) enveloping algebras and hence many different degenerations for a larger class of varieties (in the commutative case). The price to pay is that one loses control over the semigroup parameterizing the basis i.e. the exponents of the monomial basis. In general it is not known whether this semigroup is affine (though in some cases it is known that it is not normal, see the aforementioned article). In our case this is guaranteed by the fact that this semigroup is the same as that arising from the string parametrization, which is known to be affine. Thus even in the alternative approach the relation between the monomial basis and the canonical basis is essential.

4.12. Now let $\mathbb{k}$ be an arbitrary field with char $\mathbb{k}>2$, or char $\mathbb{k}>3$ if the root system of $\mathfrak{g}$ has an irreducible component of type $G_{2}$, and let $q \in \mathbb{k}^{\times}$be a nonroot of unity. There is a morphism $\mathcal{A} \longrightarrow \mathbb{k}$ induced by the assignation $v \mapsto q$, which makes $\mathbb{k}$ into an $\mathcal{A}$-bimodule. There is an algebra map $\kappa: U_{q}^{-}(\mathfrak{g}) \longrightarrow \mathbb{k} \otimes_{\mathcal{A}} U_{\mathcal{A}}^{-}$, given by sending $F_{\alpha_{i}} \in U_{q}^{-}(\mathfrak{g})$ to $1 \otimes_{\mathcal{A}} F_{\alpha_{i}}$. This map is obviously surjective, and it respects the weight decomposition of both algebras. By [Jan96, 8.24 Remark (3)], the dimension of the weight components of both algebras are given by the Kostant partition function, and hence they are equal. Thus $\kappa$ is an isomorphism.

The map $\mathbb{k} \otimes_{\mathcal{A}} V_{\mathcal{A}}(\lambda) \longrightarrow V_{q}(\lambda)$ is $U_{q}^{-}(\mathfrak{g})$-linear and sends highest weight vectors to highest weight vectors, so it is an isomorphism and the global basis of $V_{\mathcal{A}}(\lambda)$ maps to a basis of $V_{q}(\lambda)$, which we also call the global basis of $V_{q}(\lambda)$. Also, if we set $C_{\mathcal{A}}^{+}(\lambda)$ as the $\mathcal{A}$-span of $b_{\lambda}$ for $b \in \mathcal{B}(\lambda)$ we get an isomorphism $\mathbb{k} \otimes_{\mathcal{A}} C_{\mathcal{A}}^{+}(\lambda) \mapsto C_{q}^{+}(\lambda)$, with the image of the $b_{\lambda}$ forming the dual basis of the global basis of $V_{q}(\lambda)$. Thus if we set $\mathcal{O}_{\mathcal{A}}\left[G / P_{I}\right]_{w}$ to be the $\mathcal{A}$-span of $S B_{I}(w)$ inside $\mathcal{O}_{v}\left[G / P_{I}\right]_{w}$, we get that the natural map $\mathbb{k} \otimes_{\mathcal{A}} \mathcal{O}_{\mathcal{A}}\left[G / P_{I}\right]_{w} \longrightarrow \mathcal{O}_{q}\left[G / P_{I}\right]_{w}$ is an isomorphism. 
Theorem. Let $\mathbb{k}$ be any field and let $q \in \mathbb{k}^{\times}$be a nonroot of unity. Let $I \subset P$ be a set of fundamental weights, let $w \in W$, and let $\tilde{w}_{0}$ be a reduced decomposition of $w_{0}$ adapted to $w$.

The quantum Schubert variety $\mathcal{O}_{q}\left[G / P_{I}\right]_{w}$ degenerates to a quantum affine toric variety with associated semigroup $\tilde{S}_{\tilde{w}_{0}, \tilde{w}}^{I}$. In particular any quantum Schubert variety has property $\chi$, finite local dimension, the AS-Cohen-Macaulay property, and is a maximal order in its skew-field of fractions.

Proof. For each dominant integral weight $\lambda$ and each $a \in \Lambda_{\tilde{w}_{0}}(\mathcal{B}(\lambda))$, we denote by $b_{\lambda}^{a}$ the element $1 \otimes_{\mathcal{A}} b_{\lambda} \in \mathbb{k} \otimes_{\mathcal{A}} \mathcal{O}_{v}\left[G / P_{I}\right]_{w}$ where $b=\Gamma_{\tilde{w}_{0}}^{-1}(a)$. With this notation, it follows from 4.10 that for each pair of dominant integral weights $\lambda, \lambda^{\prime}$ and each $a \in \Lambda_{\tilde{w}_{0}}(\mathcal{B}(\lambda)), a^{\prime} \in \Lambda_{\tilde{w}_{0}}\left(\mathcal{B}\left(\lambda^{\prime}\right)\right), a^{\prime \prime} \in \Lambda_{\tilde{w}_{0}}\left(\mathcal{B}\left(\lambda+\lambda^{\prime}\right)\right)$ such that $a^{\prime \prime} \geq_{\text {lex }} a+a^{\prime}$, there exists $x_{a^{\prime \prime}} \in \mathcal{A}$ such that

$$
b_{\lambda}^{a} b_{\lambda^{\prime}}^{a^{\prime}}=\sum_{a^{\prime \prime} \geq a+a^{\prime}} x_{a^{\prime \prime}} b_{\lambda+\lambda^{\prime \prime}}^{a^{\prime \prime}}
$$

with $x_{a+a^{\prime}}$ a power of $q$. This implies that the basis $\left\{1 \otimes b \mid b \in S B_{I}(w)\right\}$ is a $\left(\tilde{S}_{\tilde{w}_{0}, \tilde{w}}^{I}, \leq_{\text {lex }}\right)$ basis. Thus by Proposition 3.13 we get the degeneration result.

Since $\tilde{S}_{w_{0}, I}^{w}$ is a normal semigroup, we know by Proposition 3.6 that the associated graded ring of the quantum Schubert variety has property $\chi$, finite local dimension and the AS-Cohen-Macaulay property, which $\mathcal{O}_{q}\left[G / P_{I}\right]_{w}$ inherits by Theorem 2.12 Also by Proposition 3.6 , a quantum affine toric variety whose underlying semigroup is normal is a maximal order in its ring of fractions, and it follows from [MR80, Chapitre IV, Proposition 2.1 and Chapitre V, Corollaire 2.6] that $\mathcal{O}_{q}\left[G / P_{I}\right]_{w}$ is also a maximal order.

\section{References}

[AZ94] M. Artin and J. J. Zhang, Noncommutative projective schemes, Adv. Math. 109 (1994), no. 2, 228287.

[BZo1] A. Berenstein and A. Zelevinsky, Tensor product multiplicities, canonical bases and totally positive varieties, Invent. Math. 143 (2001), no. 1, 77-128.

[BBo5] A. Björner and F. Brenti, Combinatorics of Coxeter groups, Graduate Texts in Mathematics, vol. 231, Springer, New York, 2005.

[BS98] M. P. Brodmann and R. Y. Sharp, Local cohomology: an algebraic introduction with geometric applications, Cambridge Studies in Advanced Mathematics, vol. 60, Cambridge University Press, Cambridge, 1998.

[BH93] W. Bruns and J. Herzog, Cohen-Macaulay rings, Cambridge Studies in Advanced Mathematics, vol. 39, Cambridge University Press, Cambridge, 1993.

[BGog] W. Bruns and J. Gubeladze, Polytopes, rings, and K-theory, Springer Monographs in Mathematics, Springer, Dordrecht, 2009.

[Calo2] P. Caldero, Toric degenerations of Schubert varieties, Transform. Groups 7 (2002), no. 1, 51-60.

[DCEP82] C. De Concini, D. Eisenbud, and C. Procesi, Hodge algebras, Astérisque, vol. 91, Société Mathématique de France, Paris, 1982. With a French summary. 
[FFLi7a] X. Fang, G. Fourier, and P. Littelmann, On toric degenerations of flag varieties, Representation theory-current trends and perspectives, EMS Ser. Congr. Rep., Eur. Math. Soc., Zürich, 2017, pp. $187-232$.

[FFL17b] _ Essential bases and toric degenerations arising from birational sequences, Adv. Math. 312 (2017), 107-149.

[RGS99] J. C. Rosales and P. A. García-Sánchez, Finitely generated commutative monoids, Nova Science Publishers, Inc., Commack, NY, 1999.

[GL96] N. Gonciulea and V. Lakshmibai, Degenerations of flag and Schubert varieties to toric varieties, Transform. Groups 1 (1996), no. 3, 215-248.

[Jan96] J. C. Jantzen, Lectures on quantum groups, Graduate Studies in Mathematics, vol. 6, American Mathematical Society, Providence, RI, 1996.

[JZoo] P. Jørgensen and J. J. Zhang, Gourmet's guide to Gorensteinness, Adv. Math. 151 (2000), no. 2, 313-345.

[Kas93] M. Kashiwara, The crystal base and Littelmann's refined Demazure character formula, Duke Math. J. 71 (1993), no. 3, 839-858.

[Kas95] _. On crystal bases, Representations of groups (Banff, AB, 1994), CMS Conf. Proc., vol. 16, Amer. Math. Soc., Providence, RI, 1995, pp. 155-197.

[LR92] V. Lakshmibai and N. Reshetikhin, Quantum flag and Schubert schemes, physics (Amherst, MA, 1990), Contemp. Math., vol. 134, Amer. Math. Soc., Providence, RI, 1992, pp. 145-181.

[LRo6] T. H. Lenagan and L. Rigal, Quantum graded algebras with a straightening law and the AS-CohenMacaulay property for quantum determinantal rings and quantum Grassmannians, J. Algebra $3 \mathbf{0 1}$ (2006), no. 2, 670-702.

[Lit98] P. Littelmann, Cones, crystals, and patterns, Transform. Groups 3 (1998), no. 2, 145-179.

[Lus1o] G. Lusztig, Introduction to quantum groups, Modern Birkhäuser Classics, Birkhäuser/Springer, New York, 2010. Reprint of the 1994 edition.

[MR8o] G. Maury and J. Raynaud, Ordres maximaux au sens de K. Asano, Lecture Notes in Mathematics, vol. 808, Springer, Berlin, 1980 (French).

[MRo1] J. C. McConnell and J. C. Robson, Noncommutative Noetherian rings, Revised edition, Graduate Studies in Mathematics, vol. 30, American Mathematical Society, Providence, RI, 2001. With the cooperation of L. W. Small.

[MSo5] E. Miller and B. Sturmfels, Combinatorial commutative algebra, Graduate Texts in Mathematics, vol. 227, Springer-Verlag, New York, 2005.

[NVO79] C. Năstăsescu and F. Van Oystaeyen, Graded and filtered rings and modules, Lecture Notes in Mathematics, vol. 758, Springer, Berlin, 1979.

[NVOo4] C. Năstăsescu and F. Van Oystaeyen, Methods of graded rings, Lecture Notes in Mathematics, vol. 1836, Springer-Verlag, Berlin, 2004.

[RZ12] L. Rigal and P. Zadunaisky, Quantum analogues of Richardson varieties in the grassmannian and their toric degeneration, J. Algebra 372 (2012), 293-317.

[RZ15] _ Twisted Semigroup Algebras, Alg. Rep. Theory, posted on 2015, DOI 10.1007/s10468015-9525-z.

[Sŏ̌g2] Ya. S. Soŭbel'man, On the quantum flag manifold, Funktsional. Anal. i Prilozhen. 26 (1992), no. 3, 90-92 (Russian); English transl., Funct. Anal. Appl. 26 (1992), no. 3, 225-227.

[Stu96] B. Sturmfels, Gröbner bases and convex polytopes, University Lecture Series, vol. 8, American Mathematical Society, Providence, RI, 1996.

[Weig4] C. A. Weibel, An introduction to homological algebra, Cambridge Studies in Advanced Mathematics, vol. 38, Cambridge University Press, Cambridge, 1994. 
Laurent RIGAL,

Université Paris 13, Sorbonne Paris Cité, LAGA, UMR CNRS 7539, 99 avenue J.-B. Clément, 93430 Villetaneuse, France; e-mail: rigal@math.univ-parisi3.fr

Pablo ZADUNAISKY,

Universidad CAECE, Departamento de Matemáticas. Av. de Mayo 866 - Buenos Aires, Argentina. e-mail: pzadub@dm.uba.ar 